\title{
New results on source and diffusion spectral features of Galactic cosmic rays: I $B / C$ ratio
}

\author{
D. Maurin ${ }^{1}$, R. Taillet ${ }^{1,2}$, and F. Donato ${ }^{3}$ \\ 1 Laboratoire de Physique Théorique LAPTH, 74941 Annecy-le-Vieux, France \\ 2 Université de Savoie, 73011 Chambéry, France \\ 3 Università degli Studi di Torino and INFN, Torino, Italy \\ Received 18 June 2002 / Accepted 29 July 2002
}

\begin{abstract}
In a previous study (Maurin et al. 2001), we explored the set of parameters describing diffusive propagation of cosmic rays (galactic convection, reacceleration, halo thickness, spectral index and normalization of the diffusion coefficient), and we identified those giving a good fit to the measured $B / C$ ratio. This study is now extended to take into account a sixth free parameter, namely the spectral index of sources. We use an updated version of our code where the reacceleration term comes from standard minimal reacceleration models. The goal of this paper is to present a general view of the evolution of the goodness of fit to $B / C$ data with the propagation parameters. In particular, we find that, unlike the well accepted picture, and in accordance with our previous study, a Kolmogorov-like power spectrum for diffusion is strongly disfavored. Rather, the $\chi^{2}$ analysis points towards $\delta \gtrsim 0.7$ along with source spectra index $\lesssim 2.0$. Two distinct energy dependences are used for the source spectra: the usual power-law in rigidity and a law modified at low energy, the second choice being only slightly preferred. We also show that the results are not much affected by a different choice for the diffusion scheme. Finally, we compare our findings to recent works, using other propagation models. This study will be further refined in a companion paper, focusing on the fluxes of cosmic ray nuclei.
\end{abstract}

Key words. ISM: cosmic rays

\section{Introduction}

Cosmic rays detected on Earth with kinetic energies per nucleon from $100 \mathrm{MeV} /$ nuc to $100 \mathrm{GeV} /$ nuc were most probably produced by the acceleration of a low energy galactic population of nuclei, followed by diffusion in the turbulent magnetic field. The acceleration process and the diffusion process have a magnetic origin, so that they should depend on rigidity. The rigidity dependence of the diffusion coefficient is given by quasi-linear theory as

$K(\mathcal{R})=K_{0} \beta\left(\frac{\mathcal{R}}{1 \mathrm{GV}}\right)^{\delta}$

where the parameters $K_{0}$ and $\delta$ should ideally be given by the small-scale structure of the magnetic field responsible for the diffusion. As this structure is not well observed, some theoretical assumptions must be made in order to predict $\delta$. As regards the spectrum just after acceleration, the situation is far from clear, as it depends on the details of the acceleration process. Several models give a power-law distribution (e.g. Berezhko et al. 1994; Gieseler et al. 2000)

$\frac{\mathrm{d} Q}{\mathrm{~d} p} \propto \mathcal{R}^{-\alpha}$

with a definite value for $\alpha$ which depends on the model.

Send offprint requests to: $\mathrm{R}$. Taillet,

e-mail: taillet@lapp.in2p3.fr
Most analyses of cosmic ray nuclei data assume given power-laws for the diffusion and acceleration energy dependence, so that the results partially reflect certain theoretical a priori. In this work, we try to avoid this bias by determining the quantities $\alpha$ and $\delta$ directly from the data, in particular $B / C$, for reasons exposed below.

The paper is organized as follows. We first recall the main features of our diffusion model. As a few modifications have been made since previous works, Sect. 3 is devoted to their description and justification. Then, the analysis method is described in Sect. 4 and the results are shown and discussed in Sect. 5; a comparison is eventually made with other similar works in Sect. 6.

\section{Description of the model}

This paper and its companion (Donato et al. in preparation) use the same description of cosmic ray propagation as our previous analyses (Maurin et al. 2001; Donato et al. 2001; Donato et al. 2002; Barrau et al. 2002; Maurin et al. 2002). Particles are accelerated in a thin galactic disk, from which they diffuse in a larger volume. When they cross the disk, they may interact with interstellar matter, which leads to nuclear reactions (spallations) - changing their elemental and isotopic composition and to energy losses. Interaction with Alfvén waves in the disk also leads to diffusive reacceleration. The reader is referred to Maurin et al. (2001) - hereafter Paper I - for all details, i.e. 
geometry and solutions of our two zone/three-dimensional diffusive model, nuclear parameters (nuclear grid and cross sections), energy losses terms (adiabatic, ionization and Coulomb losses), solar modulation scheme (force-field), as well as general description of the procedure involved in our fits to data (selection of a set of parameters, $\chi^{2}$ test comparison to data). In particular, though some inputs are modified (see next section), the final equation describing cosmic ray equilibrium is formally equivalent to that of Paper I (see Eq. (A13)): it is a second order differential equation in energy solved with the CrankNicholson approach (see Donato et al. 2001, Appendix B hereafter Paper II). Finally, a schematic view of our diffusion model is presented in Barrau et al. (2002) and Fig. 1 (see next section) summarizes the algorithm of our propagation code.

Some aspects of this model are formally unrealistic. First, the distribution of interstellar matter has a very simple structure: it does not take into account a possible $z$ distribution inside the disk (thin disk approximation is used instead), nor radial and angular dependence in the galactic plane. The orthoradial $\theta$ dependence would even be more important from an accurate description of the magnetic fields and the ensuing diffusion, as flux tubes are likely to be present along the spiral arms. However, this is not crucial as we are interested in effective quantities (diffusion coefficient and interstellar density) but not in giving them a "microscopic" explanation. This is why we chose to use a universal form of the diffusion coefficient, with the same value in the whole Galaxy. Finally, it is known that a fully realistic model has to take into account interactions between cosmic ray pressure, gas and magnetic pressure, i.e. magnetohydrodynamics.

The semi-analytical diffusion approach should be thought of as an intermediate step between leaky box approaches and magnetohydrodynamics simulations and is actually justified by these two very approaches: the first showed that the local abundances of charged nuclei can be roughly described by two phenomenological coefficients - the escape length and the interstellar gas density in the box. The second hints at the fact that the propagation models such as the one used here are well suited for the description of cosmic ray physics.

However, it is difficult to conclude whether these parameters are valid for other kinds of cosmic rays $\left(\mathrm{e}^{+}, \mathrm{e}^{-}\right.$, nuclei induced $\gamma$-ray production) and whether they are either meaningful but valid only locally on a few kpc scale (i.e. not in the whole Galaxy - see as an illustration Breitschwerdt et al. 2002), or meaningless but phenomenologically valid as an average description of more subtle phenomena (see as an example the discussion of the Alfvénic speed in Sect. 6.3.4).

\section{New settings}

Only a few ingredients differ from our previous analysis (Paper I). The reason for these few changes is twofold: first, we attempt to use a better motivated form of the reacceleration term; second, as the real value of the exponent in the source power-law cannot be firmly established from acceleration models - the latter being seemingly different from what is naively deduced from direct spectra measurements -, it becomes a free parameter in the present analysis.

\subsection{Transport of cosmic rays}

The starting point of all cosmic ray data analysis is the transport equation. As emphasized in Berezinskii et al. (1990), a diffusion-like equation was first obtained phenomenologically. Afterwards, the kinetic theory approach provided grounds for a consistent derivation. This transport equation reads:

$$
\begin{array}{r}
\frac{\partial f}{\partial t}-\nabla\left(K \nabla f-V_{\mathrm{c}} f\right)-\frac{\nabla \cdot V_{\mathrm{c}}}{3} \frac{1}{p^{2}} \frac{\partial}{\partial p}\left(p^{3} f\right)= \\
\frac{1}{p^{2}} \frac{\partial}{\partial p} p^{2} K_{\mathrm{pp}} \frac{\partial}{\partial p} f+\frac{\mathrm{d} Q}{\mathrm{~d} p} .
\end{array}
$$

In this equation, $f \equiv f(t, r, p)$ is the phase space distribution, $K$ is the spatial diffusion coefficient, $K_{\mathrm{pp}}$ is the momentum diffusion coefficient; both are related to the diffusive nature of the process. Finally $V_{\mathrm{c}}$ is the velocity describing the convective transport of cosmic rays away from the galactic plane. Actually, the full equation of cosmic ray transport includes other terms, such as catastrophic and spontaneous losses, secondary spallative contributions and continuous energy losses (coulombian and ionization losses). These were taken into account as described in detail in Paper I, to which the reader is referred for a complete description and references. They will not be further discussed here.

This equation can be rewritten using the cosmic ray differential density $\mathrm{d} n / \mathrm{d} E \equiv N(E)$. As the momentum distribution function is normalized to the total cosmic ray number density $\left(n=4 \pi \int \mathrm{d} p p^{2} f\right)$, we have $N(E)=(4 \pi / \beta) p^{2} f$ to finally obtain

$$
\begin{array}{r}
\frac{\partial N(E)}{\partial t}-\nabla\left[K \nabla N(E)-V_{\mathrm{c}} N(E)\right]-\frac{\left(\nabla \cdot V_{\mathrm{c}}\right)}{3} \frac{\partial}{\partial E}\left(\frac{p^{2}}{E} N(E)\right)= \\
\frac{\partial}{\partial E}\left[-\frac{\left(1+\beta^{2}\right)}{E} K_{\mathrm{pp}} N(E)+\beta^{2} K_{\mathrm{pp}} \frac{\partial N(E)}{\partial E}\right]+Q(E)
\end{array}
$$

with

$Q(E) \propto \frac{p^{2}}{\beta} \frac{\mathrm{d} Q}{\mathrm{~d} p}$.

In this paper, $K_{\mathrm{pp}}$ will be taken from the quasi-linear theory (see below).

From a theoretical point of view, the most natural choice for the energy dependence of the source term seems to be a power-law in rigidity (or momentum) for $\mathrm{d} Q / \mathrm{d} p$. This translates into $Q(E) \propto R^{-\alpha} / \beta$ in our set of equations (see Eqs. (4) and (5) above). Several different forms were used in the past because of the lack of strong evidence from observed spectra (see for example Engelmann et al. 1985; Engelmann et al. 1990). In particular, our previous analysis allowed only a rigidity dependence $Q(E) \propto R^{-\alpha}$ (for the special case $\gamma=\delta+\alpha \approx 2.8$ ). These two forms differ only at low energy and we chose to keep them both to estimate their effect on our results. As we show below, it is quite small.

Finally, different diffusion schemes lead to different forms for the energy dependence of the diffusion coefficient and the reacceleration term. Several aspects of the diffusion process are treated in Schlickeiser (2002), and we considered three alternative possibilities: (i) Slab Alfven wave turbulence, with $K_{\mathrm{A}}(p)=K_{0} \beta \mathcal{R}^{\delta}$ and $K_{\mathrm{pp}}^{\mathrm{A}} \propto V_{\mathrm{A}}^{2} p^{2} / K_{\mathrm{A}}(p)$, (ii) Isotropic fast 
magnetosonic wave turbulence, with $K_{\mathrm{F}}(p)=K_{0} \beta^{2-\delta} \mathcal{R}^{\delta}$ and $K_{\mathrm{pp}}^{\mathrm{F}} \propto V_{\mathrm{A}}^{2} p^{2} \beta^{1-\delta} \ln \left(v / V_{\mathrm{A}}\right) / K_{\mathrm{F}}(p)$, and (iii) mixture of the two last cases, $K_{\mathrm{M}}(p)=K_{0} \beta^{1-\delta} \mathcal{R}^{\delta}$ and $K_{\mathrm{pp}}^{\mathrm{M}}=K_{\mathrm{pp}}^{\mathrm{F}}$.

All results will be presented with the case (i), except in the specific discussion in Sect. 5.5.

\subsection{Summary: Updates of Paper l's formulae}

The only changes with our previous study are

- Eq. (19) of Paper I is replaced by

$$
b_{\text {loss }}^{j}(E)=\left\langle\frac{\mathrm{d} E}{\mathrm{~d} t}\right\rangle_{\text {Ion }}+\left\langle\frac{\mathrm{d} E}{\mathrm{~d} t}\right\rangle_{\text {Coul }}+\left\langle\frac{\mathrm{d} E}{\mathrm{~d} t}\right\rangle_{\text {Adiab }}+\left\langle\frac{\mathrm{d} E}{\mathrm{~d} t}\right\rangle_{\text {Reac }},
$$

where

$$
\langle\mathrm{d} E / \mathrm{d} t\rangle_{\text {Reac }}=\frac{\left(1+\beta^{2}\right)}{E} K_{\mathrm{pp}} .
$$

- Eq. (A13) of Paper I (second order differential equation to solve) reads now - we use the same notations -

$$
A_{i}^{j} N_{i}^{j}(0)=\bar{Q}^{j}-2 h \frac{\partial}{\partial E}\left\{b_{\text {loss }}^{j}(E) N_{i}^{j}(0)-\beta^{2} K_{\mathrm{pp}} \frac{\partial}{\partial E} N_{i}^{j}(0)\right\}
$$

with

$$
K_{\mathrm{pp}}=\frac{h_{\mathrm{reac}}}{h} \times \frac{4}{3 \delta\left(4-\delta^{2}\right)(4-\delta)} V_{\mathrm{a}}^{2} p^{2} / K(E) .
$$

In our model, $h_{\text {reac }} \equiv h$, but it has to be kept in mind that a possible reinterpretation of $V_{\mathrm{a}}$ is always possible (see Sect. 6) as long as $h_{\text {reac }} \ll L$ (this condition is necessary for the solution to be valid).

- As regards the source spectra, two forms (hereafter type (a) and (b)) are used instead of Eq. (9) of Paper I

$$
\begin{aligned}
& a-Q(E) \propto \frac{1}{\beta} R^{-\alpha} \\
& b-Q(E) \propto R^{-\alpha}
\end{aligned}
$$

where $R$ is the rigidity and $\alpha$ a universal slope of spectra for all nuclei heavier than helium.

\section{Runs and selection method}

The analysis presented here is the natural continuation of the work presented in Paper I. It is more general and it encompasses its results as a five-dimensional subset of the sixdimensional space scanned here.

\subsection{The six free parameters of the study}

The six parameters of this study are: the spectral index of sources $\alpha$, the normalization $K_{0}$ and spectral index $\delta$ of the diffusion coefficient, the height of the diffusive halo $L$, the Galactic convective wind speed $V_{\mathrm{c}}$ and the Alfvénic speed $V_{\mathrm{a}}$. They are included in our code as follows (see Fig. 1 for a sketch of the procedure): for a given set of parameters, source abundances of all nuclei (i.e. primaries and mixed nuclei) are adjusted so that the propagated top of atmosphere fluxes agree with the data at $10.6 \mathrm{GeV} / \mathrm{nuc}$ (see Paper I). We remind that for $B / C$ ratio, we checked that starting the evaluation of fluxes from Sulfur is sufficient (heavier nuclei do not contribute significantly to this ratio). Top of atmosphere fluxes are deduced from interstellar fluxes using the force field modulation scheme (see Paper I and references therein). The resulting $B / C$ spectrum is then compared to the data (see below) and a $\chi^{2}$ is computed for the chosen set of parameters.

This procedure is very time consuming. Even when the location of $\chi^{2}$ minima in the six-dimensional parameter space are known, more than $2 \times 10^{6}$ configurations are needed to have a good sampling of the regions of interest, for a given form of the source term energy dependence.

\section{2. $\chi^{2}$ criterion of goodness}

As in our previous analysis, we have computed the quantity

$\chi^{2}=\sum_{i} \frac{\left((B / C)_{i, \text { exp }}-(B / C)_{i, \text { model }}\right)^{2}}{\sigma_{i, \text { xp }}^{2}}$

where the sum runs over 26 experimental values from HEAO-3 (Engelmann et al. 1990) with energies ranging from $620 \mathrm{MeV} /$ nuc to $35 \mathrm{GeV} / \mathrm{nuc}$ (as in Paper I). In general, if the experimental set-up is such that the measured (experimental) values differ from the "real" values by a quantity of zero mean (non biased) with a given probability distribution, then the value of $\chi^{2}$ gives a quantitative estimate of the probability that the model is appropriate to describe the data. However, this condition is probably not fulfilled for HEAO-3, as for some measured quantity, the quoted errors $\sigma_{i, \text { exp }}^{2}$ are much smaller (e.g. oxygen fluxes) or much larger (e.g. sub-Fe/Fe ratio) than the dispersion of data itself. For this reason, it is meaningless to associate a likelihood to given $\chi^{2}$ values. Instead, in Paper I we decided that models giving $\chi^{2}$ less than some value $\chi_{0}^{2}$ were "good fits" while the others were "poor fits". In this paper, no cut is applied and all the models, whatever the value of $\chi^{2}$, are shown in the figures.

\section{Results}

\subsection{Subset 1: Fixed measured spectral index

$$
\gamma=\alpha+\delta \equiv 2.8
$$

In this section we present the results obtained for source spectra of the form $Q(E) \propto R^{-\alpha} / \beta$ and diffusion coefficient $K=$ $K_{0} \beta \mathcal{R}^{\delta}$. At sufficiently high energies, spallations and energetic changes are irrelevant and the measured fluxes can be considered as a mere result of acceleration and diffusion (see for example Maurin et al. 2002). In this case, the observed spectrum is proportional to $\mathcal{R}^{-\gamma}$ with $\gamma \equiv \alpha+\delta$. In this section, we focus on the situation $\gamma=2.8$, corresponding to the spectral index of the measured Boron progenitor fluxes. Actually, Wiebel-Sooth et al. (1998) analysed data from several experiments and derived smaller values. In Paper I, we found that the Oxygen flux measured by HEAO-3 would be more compatible with our diffusion model for a higher $\gamma$, namely 2.8 instead of 2.68. Anyway, we are more interested in the trends in the variation of other 


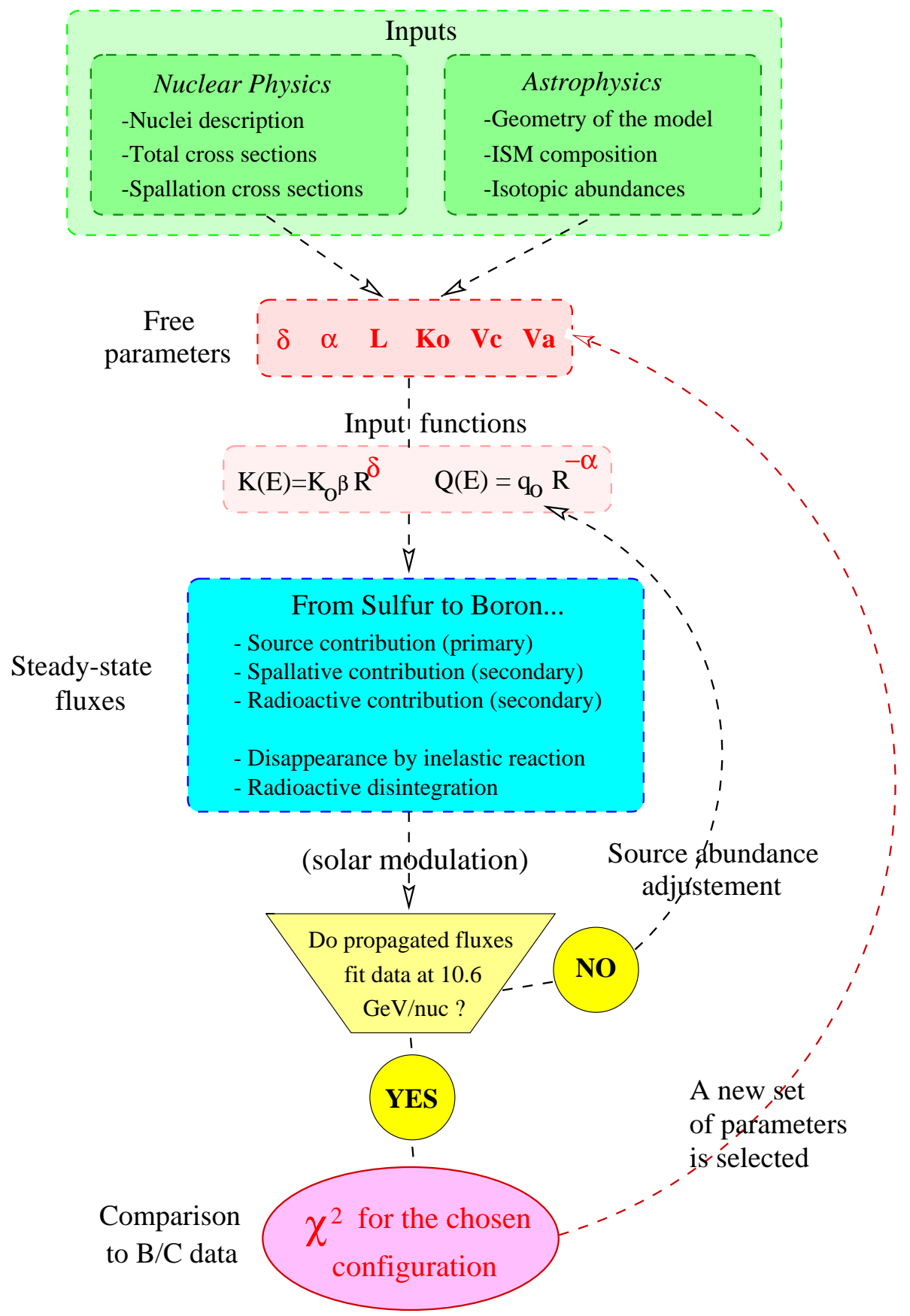

Fig. 1. Diagrammatic representation of the various steps of the propagation code.

parameters for a fixed value of $\gamma$ than in this precise numerical value. The other cases will be treated in the following sections.

We also set the halo thickness $L$ to $6 \mathrm{kpc}$, leaving us with four free parameters $\left(\delta, K_{0}, V_{\mathrm{a}}\right.$ and $\left.V_{\mathrm{c}}\right)$. All curves depicted in Fig. 2 correspond to one-dimensional cuts through the absolute $\chi^{2}$ minimum (for a given $\delta$, the three different cuts justify the fact that we are located in a minimum). In the upper panel of Fig. 2, we plot the values of the $\chi^{2}$ as a function of $K_{0} / L$, for different values of $\delta$ (and the corresponding $\alpha=$ $2.8-\delta$ ). The best fits are obtained for $\delta \sim 0.8-0.9$, far from the Kolmogorov spectrum $(\delta=1 / 3)$. We found a quite similar result in Paper I, where the same assumptions on $\gamma$ were made but with a different choice for the source spectrum, $Q(E)=\mathcal{R}^{-\alpha}$.
The fit is best for values of the diffusion coefficient normalization $K_{0} \sim 6 \times 10^{-3} \mathrm{kpc}^{2} \mathrm{Myr}^{-1}$, yielding the value $\chi^{2} \sim 40$ (giving $\chi_{\mathrm{r}}^{2} \sim 1.8$ ). For a Kolmogorov spectrum, the minimum $\chi^{2}$ is almost twice this value. Leaving aside any statistical interpretation of the analysis, we can observe that for greater $\delta$, the minima of $\chi^{2}$ are obtained for smaller $K_{0} / L$ (or $K_{0}, L$ being set to $6 \mathrm{kpc}$ ) and versa-vice. This can be understood as at a sufficiently high energy $E_{\text {thresh }}$, diffusion is the sole remaining influencial parameter and, for the flux to be unchanged with various $\delta$, one need to satisfy roughly the relation $K_{0} \times E_{\text {thresh }}^{\delta} \approx$ cte (this will also explain why type (a) and type (b) source spectra give similar $K_{0}$, see below). 

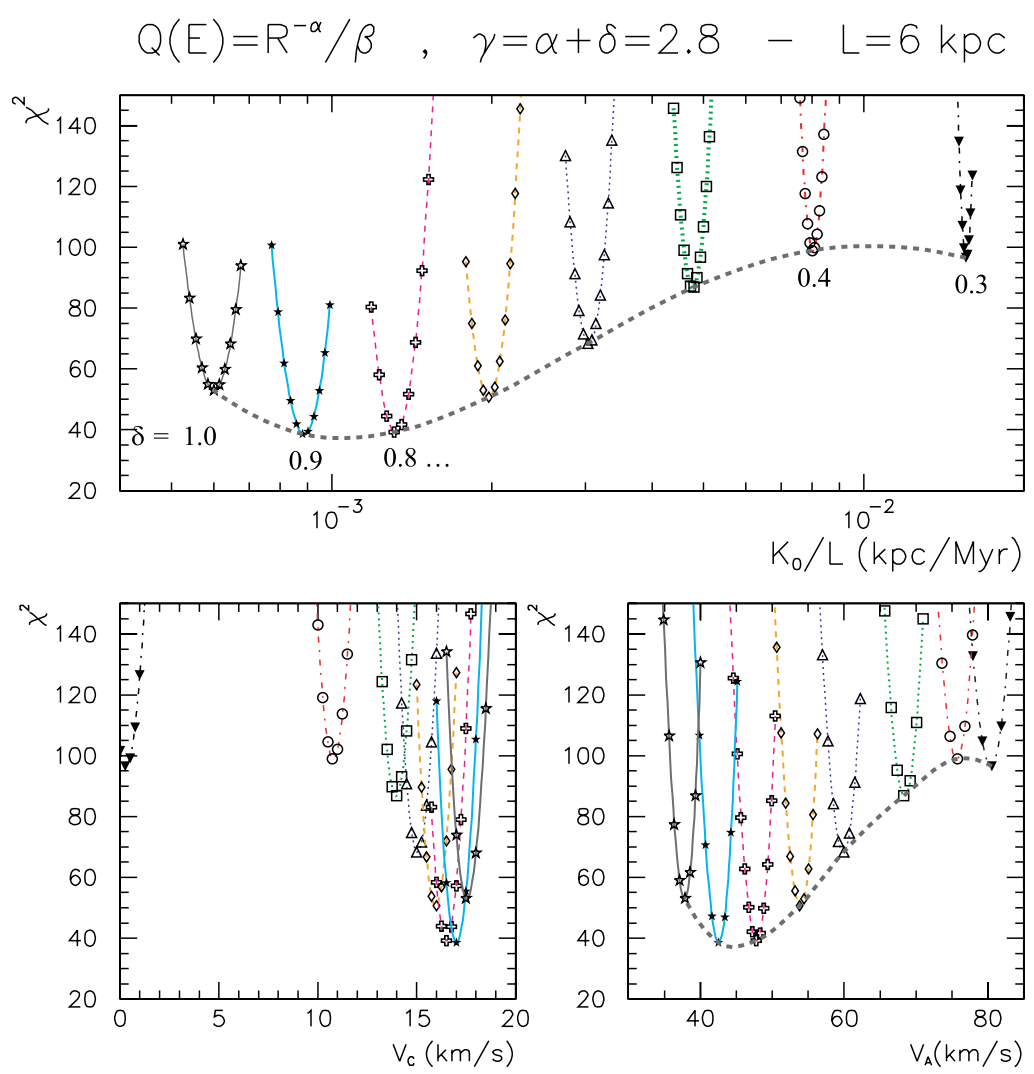

Fig. 2. Evolution of the $\chi^{2}$ value for various combination of parameters. All curves are for type (a) spectra $\left(R^{-\alpha} / \beta\right)$ with $\gamma \equiv \delta+\alpha=2.8$ and the halo size is fixed to $L=6 \mathrm{kpc}$. Each curve shows one-dimensional cuts in the $K_{0} / L$ (upper panel), $V_{\mathrm{c}}$ (left lower panel) or $V_{\mathrm{a}}$ (right lower panel) direction of the 3-dimensional $\chi^{2}$ hyper-surface. In the upper panel $\delta$ is varied from 1.0 to 0.3 and, in the lower panels the same symbols to indicate $\delta$ are conserved. Each curve gives the absolute minimum for the parameter on the abscissa axis, $L$ being fixed to $6 \mathrm{kpc}$ (similar curves with slightly different minima are obtained for other $L$ values).

In the lower panels we present two cuts in the two other directions, namely in the $V_{\mathrm{c}}$ and $V_{\mathrm{a}}$ directions. The first one tells us that except for the special case $\delta=0.3$ for which the $\chi^{2}$ curve skips to null $V_{\mathrm{c}}, B / C$ is fitted with $V_{\mathrm{c}}$ between 10 and $20 \mathrm{~km} \mathrm{~s}^{-1}$. The best $\chi^{2}$ are for convective velocity around $V_{\mathrm{c}} \sim 16-18 \mathrm{~km} \mathrm{~s}^{-1}$. For $V_{\mathrm{c}} \lesssim 15 \mathrm{~km} \mathrm{~s}^{-1}$ (and $\delta \lesssim 0.6$ ) the goodness of the fit quickly decreases. We can see that when $\delta$ is around $0.4-0.3$, the $B / C$ ratio becomes very sensitive to the $V_{\mathrm{c}}$ values. It appears that when $\delta$ is decreased, a good fit is maintained provided that $V_{\mathrm{c}}$ is also lowered. This is possible down to $\delta \sim 0.4$ for which the best value for $V_{\mathrm{c}}$ is zero. For lower $\delta$, the previous trade-off cannot be achieved (as $V_{\mathrm{c}}$ must be positive for the galactic wind to be directed outwards) and no good fit is possible.

The right panel shows the $\chi^{2}$ curves as functions of the Alfvén velocity. The minimization procedure always yields a $V_{\mathrm{a}}$ far different from zero. Good fits are obtained for values of $V_{\mathrm{a}} \sim 40-50 \mathrm{~km} \mathrm{~s}^{-1}$.

In each of the explored directions, the $\chi^{2}$ curves are very narrow: the diffusion model leads to meaningful and interpretable values for all the physical, free parameters. Similar results, with slightly different values for the minima, are obtained for the other values of $L$ in the range $1 \leq L \leq 15 \mathrm{kpc}$.
In Fig. 3 we present the results for the same analysis for different values of the halo thickness $L$ and considering also the form (b) for the source spectra, i.e. $Q(E) \propto R^{-\alpha}$. The total spectral index $\gamma$ is still set to 2.8. The left panel reports the $\chi^{2}$ as functions of $K_{0} / L$, for different values of $\delta$ and $L$, and for both types of source spectra. We see that the choice (b) globally improves the fit, and the favoured range for $\delta$ is now $\delta \gtrsim 0.4$ (whereas $\delta \gtrsim 0.7$ for choice (a)). At fixed $\delta$ and $L$, the absolute minima for both choices correspond to very similar values of $K_{0} / L$. We can also notice that type (a) spectra are, for the higher $\delta$, more sensitive to variations of $L$.

In the right panels we show a cut in the $V_{\mathrm{c}}-V_{\mathrm{a}}$ plane. For both type (a) and (b) spectra, $\delta=0.3$ yields a null value for the convective wind. Type (a) spectra give a little bit higher $V_{\mathrm{c}}$. At fixed $\delta$, the variation of $L$ has almost no effect on $V_{\mathrm{c}}$, while it is strongly correlated with the increase of $V_{\mathrm{a}}$.

\subsection{Subset 2: $\delta=0.6$, new features from $\alpha$ variation}

In this section we discuss the results obtained when the in$\operatorname{dex} \alpha$ is varied between 1.3 and $2.5, \delta$ being set to a given value $\delta=0.6$ which has been extensively used in the literature.

Figure 4 corresponds to the previous Fig. 2. In the left panel we observe that a large variation of the index $\alpha$ has a slight 
Best $\chi^{2}$ for various $\delta$ and L combinations (fixed $\gamma=2.8$ )
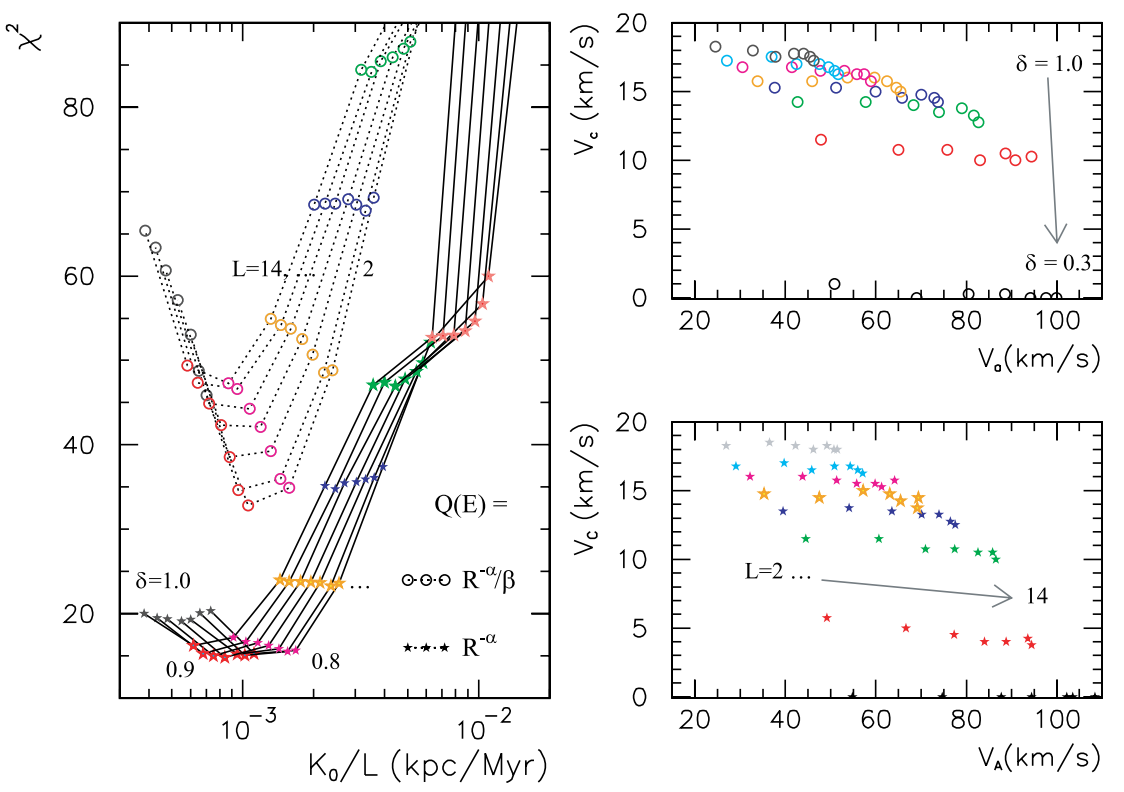

Fig. 3. Left panel: evolution of the best $\chi^{2}$ value with $K_{0} / L$ for various $\delta$ (1.0 to 0.3 , from left to right) at a fixed $\gamma=2.8$. Each curve correponds to a given halo size $L$ from $14 \mathrm{kpc}$ to $2 \mathrm{kpc}$. Right panel: the same best $\chi^{2}$ values are presented versus $V_{\mathrm{c}}$ and $V_{\mathrm{a}}$. In both panels, empty circles correspond to type (a) spectra and stars to type (b) spectra.

effect on the normalization of the diffusion coefficient $K_{0}$, which stays around an average value $K_{0} / L \sim 0.0032 \mathrm{kpc} \mathrm{Myr}^{-1}$ for $L=6 \mathrm{kpc}$. Evolution of the absolute $\chi^{2}$ minimum is also far less sensitive to $\alpha$ than $\delta$ (see previous section). However, for $\alpha \gtrsim 2.2$ the fit to the data is poor and a global power $\gamma \gtrsim 2.8$ at $\delta=0.6$ is excluded.

The lower panels represent a cut in the $V_{\mathrm{c}}$ and $V_{\mathrm{a}}$ directions. We can observe that the minimization procedure always drives the minima towards convective velocities between 12 and $16 \mathrm{~km} \mathrm{~s}^{-1}$, the least $V_{\mathrm{c}}$ being obtained for the smallest $\alpha$. This range is again very narrow. Similarly, reacceleration is needed to fit data and the minima of the $\chi^{2}$ are obtained for $V_{\mathrm{a}}$ between 55 and $75 \mathrm{~km} \mathrm{~s}^{-1}$. Towards this lower limit, $\chi^{2}$ is high and the model cannot confidently reproduce observations.

When $\delta$ is fixed, we can conclude that a variation in the power of the type (a) source spectrum does not strongly act on the evolution of $K_{0}, V_{\mathrm{c}}$ and also $V_{\mathrm{a}}$. This can be also easily understood: forgetting for a while energy gains and losses, we see from diffusion equation solutions (the same behavior occurs in leaky box models) that the source term can be factorized so that secondary to primary ratios finally do not depend on $Q(E)$, i.e. are independent of $\alpha$. Once again, the absolute minimum is identified by a steep $\chi^{2}$ in these three directions.

In Fig. 5 we present the results for $\delta=0.6$, and for both type (a) and (b) source spectra, to focus on the evolution of $L$ and $\alpha$. The left panel tells us that the evolution of the halo thickness from 2 to $14 \mathrm{kpc}$, at fixed $\alpha$ (in other words, at fixed $\gamma=\alpha+0.6)$ does not change the goodness of the fit. Only a slight modification in $K_{0} / L$ is required in order to recover the same $B / C$ flux ratio. Type (b) source spectra reproduce quite well the data for all the explored parameter space. On the contrary, the better theoretically motivated type (a) spectra cannot reproduce observations for $\alpha \gtrsim 2.2$ if $\delta=0.6$. Since at high energies the two source spectra are equivalent, we must conclude that it is the low energy part of $B / C$ which is responsible for such a discrimination.

The right panels show the absolute minima in the $V_{\mathrm{c}}-V_{\text {a }}$ plane. Both spectra require non-null reacceleration and convection. Even more so, the selected values reside in the narrow interval for $V_{\mathrm{c}}$, i.e. $V_{\mathrm{c}} \sim 10-15 \mathrm{~km} \mathrm{~s}^{-1}$ and between 40 and $90 \mathrm{~km} \mathrm{~s}^{-1}$ for the Alfvén velocity.

\subsection{Subset 3: $\alpha=2.0$, standard acceleration}

Figure 6 describes the results of the analysis done assuming type (a) source spectra, with fixed index $\alpha=2.0$ and $L=6 \mathrm{kpc}$. A consensus seems to emerge in favor of values $\alpha \approx 2.0$ (see Drury et al. 2001 and references therein), close to the index given by primeval acceleration models, but any other value would be fine for the purpose of this section. In the upper panel $\delta$ has been varied between 1.0 and 0.3 , and the figure shows the evolution of the $\chi^{2}$ with respect to $K_{0} / L$. As in Fig. 2 and, at variance with Fig. 4, the minima correspond to $K_{0} / L$ spanning over almost two orders of magnitude. It is the modification of the power-law in the diffusion coefficient - and not in the source spectrum - that significantly acts on $K_{0}$. Once again, the Kolmogorov spectrum is disfavoured: in this case it is obvious that the calculated flux ratio would be too hard. The best fits are obtained for $\delta \sim 0.6-0.9$.

The lower panels show the cuts in the $V_{\mathrm{c}}$ and $V_{\mathrm{a}}$ directions. The left one tells us that for smaller $\delta$, the preferred convective velocities are smaller (and the best $\chi^{2}$ is larger), down to 

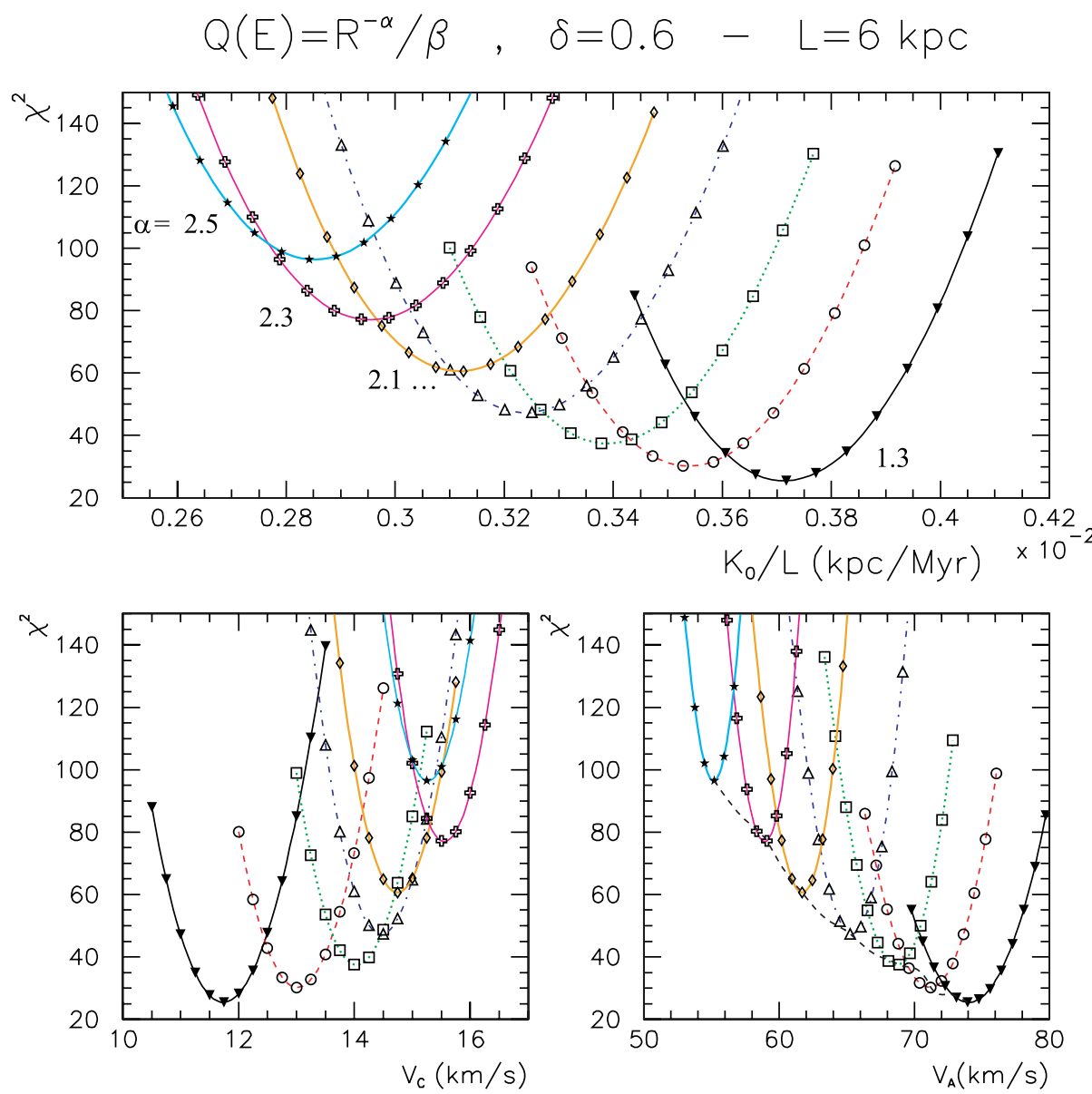

Fig. 4. Same as in Fig. 2 (type (a) spectra, $L=6 \mathrm{kpc}$ ), but for a fixed $\delta=0.6$.

Best $\chi^{2}$ for various $\alpha$ and L combinations (fixed $\delta=0.6$ )
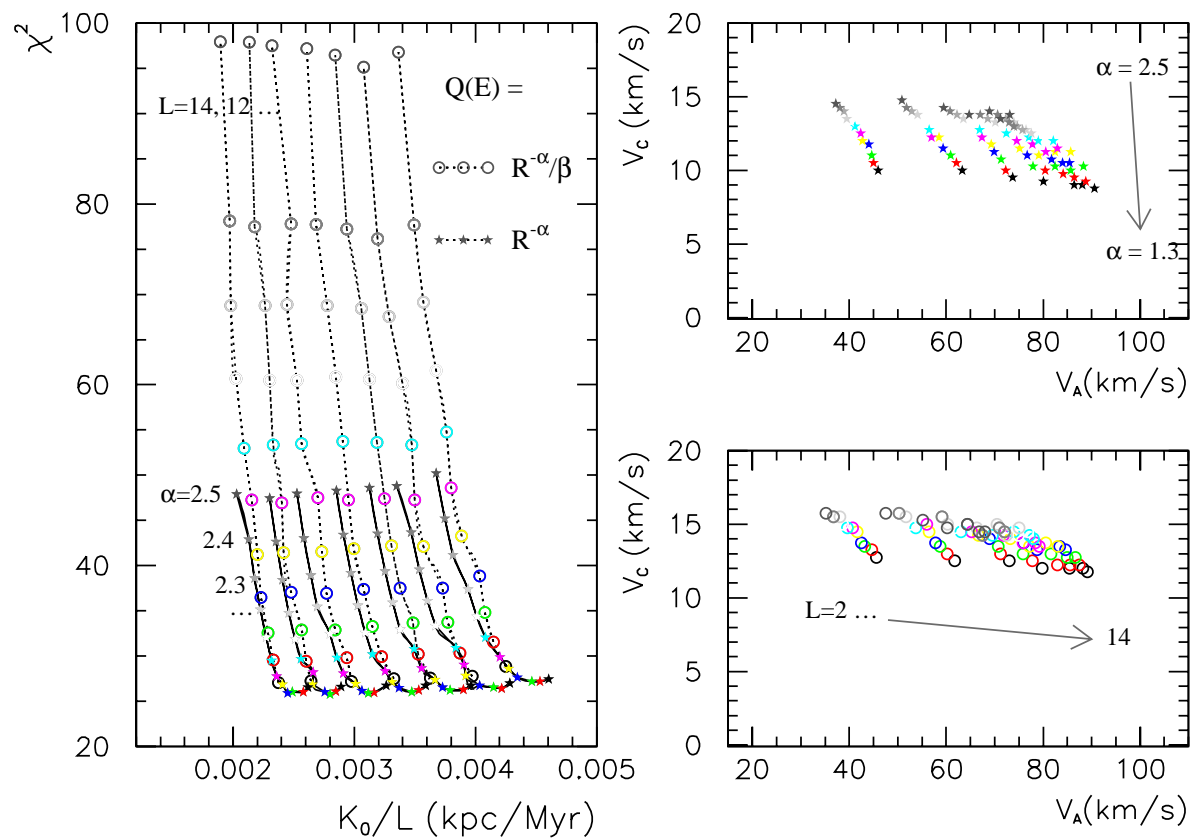

Fig. 5. Same as in Fig. 3 but for a fixed $\delta=0.6$. 

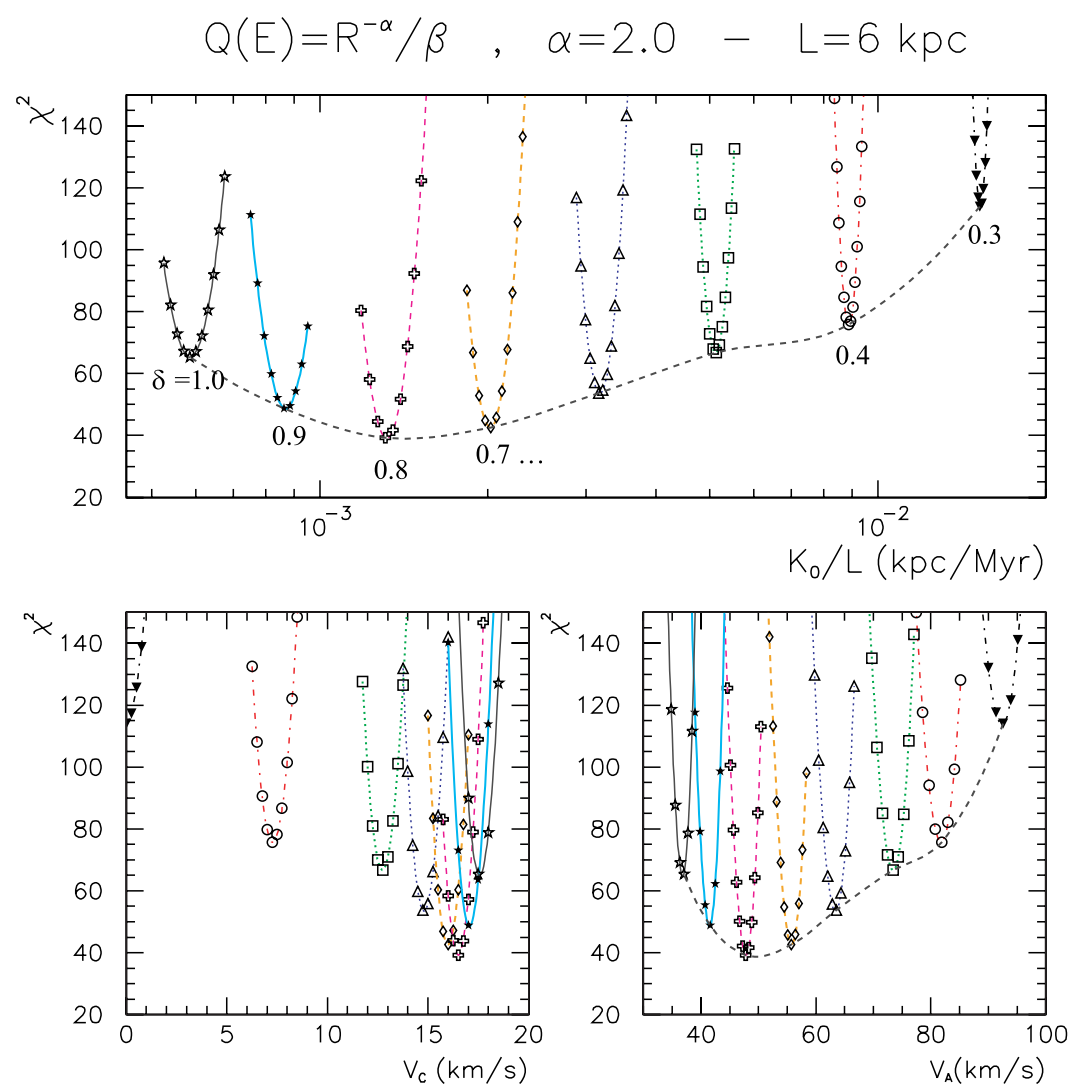

Fig. 6. Same as in Fig. 2 (type (a) spectra, $L=6 \mathrm{kpc}$ ), but for a fixed $\alpha=2.0$.

$\delta=0.3$ for which a no-convection model is prefered, with a bad $\chi^{2}$. The best fits are obtained for $V_{\mathrm{c}}$ around $15-18 \mathrm{~km} \mathrm{~s}^{-1}$. In the right panel we can notice, again, that only models with reacceleration have been chosen by the minimization procedure. Lower $\delta$ point to higher $K_{0} / L$ and $V_{\text {a }}$ values and lower $V_{\mathrm{c}}$. The same trend is recovered in the other cases treated above. Reacceleration and convection act, in a certain sense, in competition, even if data always give preference to a combined effect rather than their absence.

This trend (the smaller $\delta$, the larger $K_{0}$, or equivalently $K_{0} / L$ as $L$ is constant in the above figures) was already mentioned in Sect. 5.1. Actually, as we will see in Sect. 6, the correlation between $K_{0} / L$ and $V_{\mathrm{c}}$ is more properly explained by virtue of Eq. (17) so that the evolution of $V_{\mathrm{c}}$ is fixed by the evolution of the two other free parameters, i.e. $K_{0} / L$ and $\delta$. As regards $V_{\mathrm{a}}$, it only appears in Eq. (9). A rough estimation can be inferred using power-laws $K(E) \propto E^{\delta}$ and $N^{j}(E) \propto E^{-(\alpha+\delta)}$ in Eqs. (4) and (9):

$$
\frac{\mathrm{d}}{\mathrm{d} E}\left\{\frac{V_{\mathrm{a}}^{2} E}{K_{0} E^{\delta}} E^{-(\alpha+\delta)}+\frac{V_{\mathrm{a}}^{2} E^{2}}{K_{0} E^{\delta}}(\alpha+\delta) E^{-(\alpha+\delta-1)}\right\} .
$$

One finally obtains that the term for energetic redistributions evolves as $(3+\delta)(1+2 \delta) N(E) \times V_{\mathrm{a}}^{2} / K(E)$ for $\alpha=2$. Hence, from the above argument, when $\delta$ is decreased, $K_{0}$ is adjusted so that $K(E)$ and $N(E)$ remain grossly the same. However, for the above expression to be constant, $V_{\mathrm{a}}$ must be increased; this is the trend we observe.
In Fig. 7 we show the effect of varying the halo thickness when the source spectral index is fixed to 2.0 and all the other free parameters are scanned. Again, type (b) spectra reproduce better the data. When $L$ is varied between 14 and $2 \mathrm{kpc}$, this may modify the chosen $K_{0} / L$ by a factor of two. The right panels tell us that the influence of $L$ on $V_{\mathrm{a}}$ is to double its value when $L$ is varied from its minimum to its maximum value. On the contrary, the effect on $V_{c}$ is almost null. The situation for $V_{\mathrm{c}}$ and $V_{\mathrm{a}}$ is very similar to the one discussed in the two above cases, when $\gamma$ and then $\alpha$ were fixed. Indeed, looking carefully at the above figures, we recover the same effect also for $K_{0} / L$, at fixed $\alpha+\delta$. Again, the behaviour of $V_{\mathrm{c}}$ can be understood but cannot be simply explained. Conversely, neglecting $V_{\mathrm{c}}$ in the asymptotical formula, one can see that when $L$ increases, $K_{0}$ must increase (as can be checked in the left panel). Moreover, it can be seen from the form of $K_{\mathrm{pp}}$ that $V_{\mathrm{a}}$ increases as the square root of $K_{0}$ when $\delta$ is fixed (see right lower panel).

\subsection{The whole set: Final results}

We know present the result of the full analysis, in which all the parameters are varied. Figure 8 shows the evolution of the $\chi^{2}$ in the $\delta$ and $\gamma=\delta+\alpha$ plane for different values of $L$. We can see that, at fixed type (a) or (b) spectra, a change in the halo height $L$ has almost no effect on the best $\chi^{2}$ surface. Generally, high values for $\delta$ are preferred and, a Kolmogorov regime for the spatial diffusion coefficient is strongly disfavoured over all 
Best $\chi^{2}$ for various $\delta$ and $L$ combinations (fixed $\alpha=2.0$ )
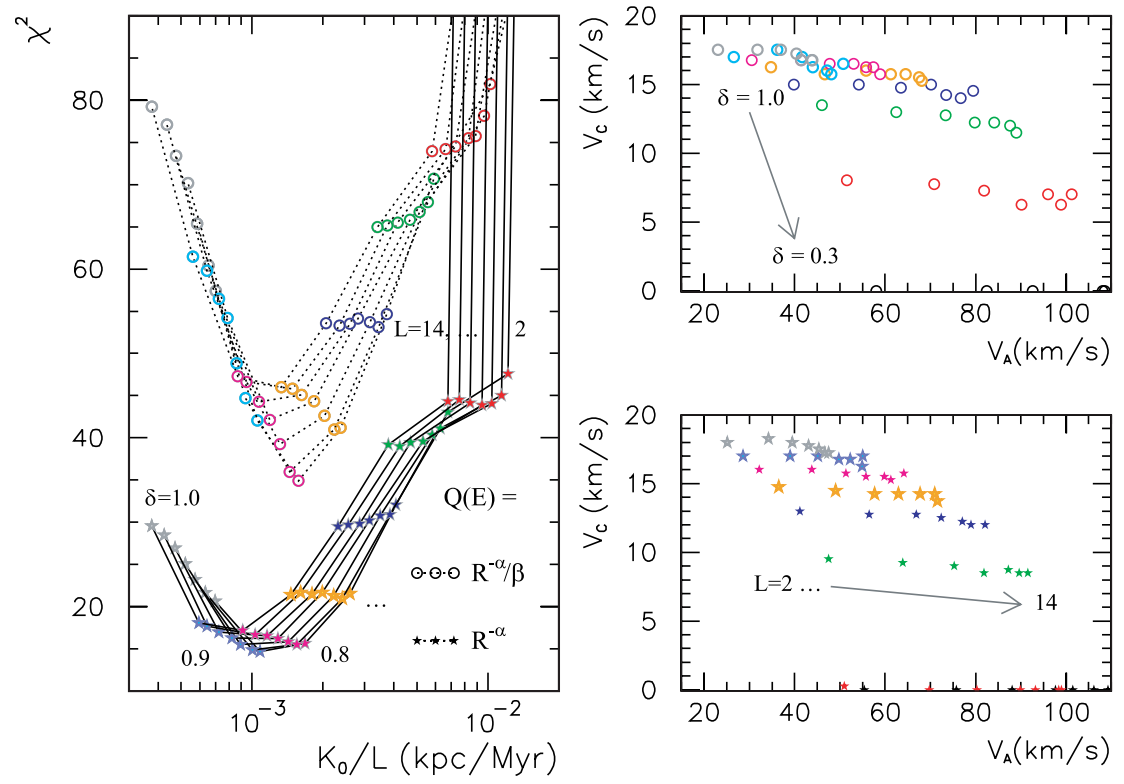

Fig. 7. Same as in Fig. 3 but for a fixed $\alpha=2.0$.

the parameter space. More precisely, type (b) spectra point towards a band defined by $\delta \sim 0.8$ in the $\delta-\gamma$ plane, whereas the type (a) spectra gives the additional constraint $\gamma \lesssim 2.8$ (see Fig. 8).

In Fig. 9 we show the preferred values of the three remaining diffusion parameters $K_{0}, V_{\mathrm{c}}$ and $V_{\mathrm{a}}$, for each best $\chi^{2}$ in the $\delta-\gamma$ plane, when $L$ has been fixed to $6 \mathrm{kpc}$. The two upper panels show that the evolution of $\alpha$ does not affect $K_{0}$. On the other hand, as already noticed, we clearly see the (anti)correlation between the two parameters $K_{0}$ and $\delta$ entering the diffusion coefficient formula, giving the same normalization at high en$\operatorname{ergy}\left(K_{0} \times E_{\text {thresh }}^{\delta} \approx c t e\right)$. Almost the same numbers are obtained for type (a) and (b) spectra. $K_{0}$ spans between 0.003 and $0.1 \mathrm{kpc}^{2} \mathrm{Myr}^{-1}$. We will discuss in the following sections how these results can be compared to the literature.

The middle panels show the values for the convective velocity. Only very few configurations include $V_{\mathrm{c}}=0$, always when $\delta=0.3$, for both types of source spectra. The value of $V_{\mathrm{c}}$ increases with $\delta$. For type (a) spectra, increasing $\gamma$ and $\delta$ at the same time makes $V_{\mathrm{c}}$ change its trend. As remarked previously, the effect of Galactic wind is more subtle since it acts at intermediate energies and is correlated with all the other diffusion parameters through the numerous terms of the diffusion equation.

The lowest two panels show the influence of $V_{\mathrm{a}}$. We recover a correlation similar to the one discussed for $K_{0}$ (see Eq. (13)). The Alfvén velocity doubles from $\delta=1.0$ to 0.3 , whereas it is almost unchanged by a variation in the parameter $\gamma$ (or equivalently $\alpha$ ).

All the three analysed parameters (i.e. $K_{0}, V_{\mathrm{c}}$ and $V_{\mathrm{a}}$ ) behave very similarly with respect to a change in the source spectrum from type (a) to type (b). It can be explained as the influence on the primary and secondary fluxes can be factored out (see Sect. 5.2) if energy changes are discarded (their effect is actually small on the derived parameters). Existing data on $B / C$ do not allow us to discriminate clearly between these two shapes for the acceleration spectrum. This goal could be reached by means of better data not only for $B / C$ but also for primary nuclei (Donato et al., in preparation).

\subsection{Other diffusion schemes}

As discussed in Sect. 3.1, we tested three different diffusion schemes, with three different forms for the diffusion coefficient. Most results are basically insensitive to the choice of this form. In particular, the figures corresponding to Fig. 9 are almost identical to the case presented above, so that they will not be reproduced here. Figure 10 displays the $\chi^{2}$ as a function of $\delta$ and $\gamma$. The values of $\chi^{2}$ are slightly different in the three cases, but the general trend is the same, and all the previous conclusions still apply.

\subsection{Sub-Fe/Fe ratio}

In an ideal situation in which we had very good and consistent data on $B / C$ and sub- $\mathrm{Fe} / \mathrm{Fe}$ ratios, the best attitude would be to make a statistical analysis of the combined set of data. Unfortunately, this is not currently the case. We consider two ways to extract information from the Sub-Fe/Fe data. First, as a check, we compare the sub-Fe/Fe ratio predicted by our model - using the parameters derived from our above $B / C$ analysis - with data from the same experiment. Second, we search directly the minimum $\chi_{\mathrm{Fe}}^{2}$ of the sub-Fe/Fe ratio, with no prior coming from $B / C$. As previously emphasized (see Sect. 4.2), 


$$
Q(E)=R^{-\alpha} / \beta \quad Q(E)=R^{-\alpha}
$$
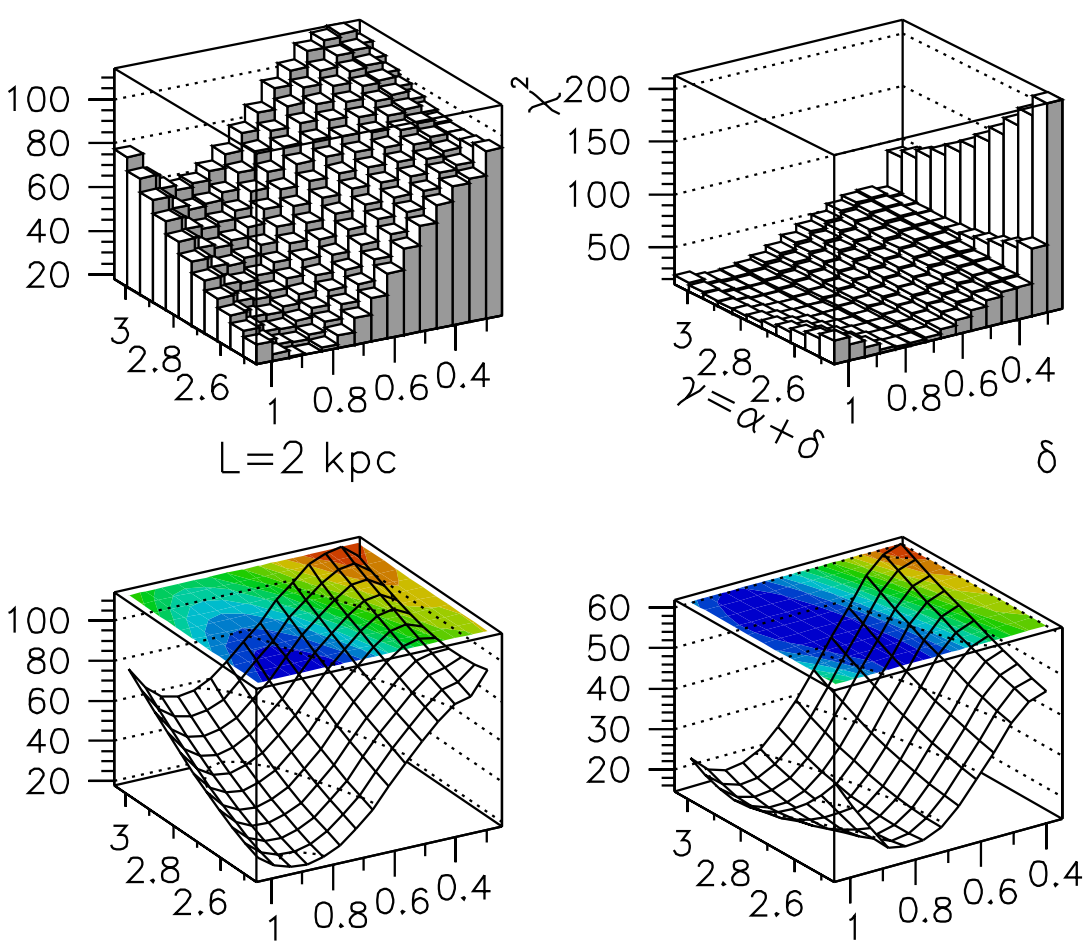

$$
L=6 \mathrm{kpc}
$$
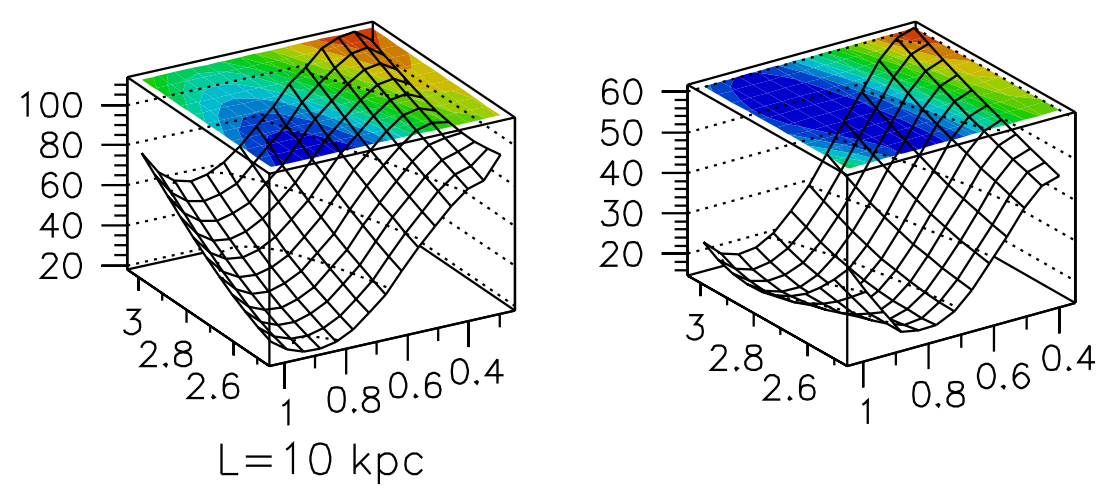

Fig. 8. Best $\chi^{2}$ values for various $L(2,6$ and $10 \mathrm{kpc})$ in the plane $\delta-\gamma$. Left histograms are type (a) spectra and right histograms type (b). Notice that for right histograms, only the upper figure displays the values $\delta=0.3$ and $\delta=0.35$. They have been omitted in the two remaining figures to gain contrast (for any $L$, these configurations have $\chi^{2} \gtrsim 100$ ). Assuming $L=6 \mathrm{kpc}$, type (a) source spectra give a best value $\chi_{\text {best }}^{2}=17.8$ for $\alpha=1.65$ and $\delta=0.85$ whereas type (b) gives $\chi_{\text {best }}^{2}=14.6$ for $\alpha=1.95$ and $\delta=0.85$. These were obtained with 26 data points.

this procedure is more hazardous since the statistical significance of the sub-Fe/Fe data is far from clear.

\subsubsection{Using $B / C$-induced parameters to derive $\chi_{B / C \rightarrow \mathrm{Fe}}^{2}$}

For each set of diffusion parameters giving a good fit to the observed $B / C$ ratio, the sub-Fe/Fe ratio can be computed and compared to the values measured by HEAO-3. This is not as straightforward as in the $B / C$ case because although $\mathrm{Sc}, \mathrm{Ti}$ and $\mathrm{V}$ - that enter in the sub-Fe group (as combined in data here) - are pure secondaries, some of the species intermediate between sub-Fe and Fe, contributing to the sub-Fe flux, are mixed species (i.e. $\mathrm{Cr}, \mathrm{Mn}$ ). As a consequence, all the primary contributions were adjusted so as to reproduce the sub-Fe/Fe ratio at $3.35 \mathrm{GeV} /$ nuc. The sub-Fe/Fe spectra are not steep enough at high energy, so that normalization at $10.6 \mathrm{GeV}$ (i.e. as for $B / C$ ) would have led to less good fits. We emphasize that to perform this normalization of secondary-to-primary is equivalent to making an assumption about the elemental composition of the sources, which is usually deduced from secondaryto-primary ratios. A different choice would slightly shift the normalization of sub-Fe/Fe ratio without affecting much our conclusions.

Figure 11 displays the $\chi_{B / C \rightarrow \mathrm{Fe}}^{2}$ values obtained when the diffusion parameters giving a good fit to $B / C$ are used 

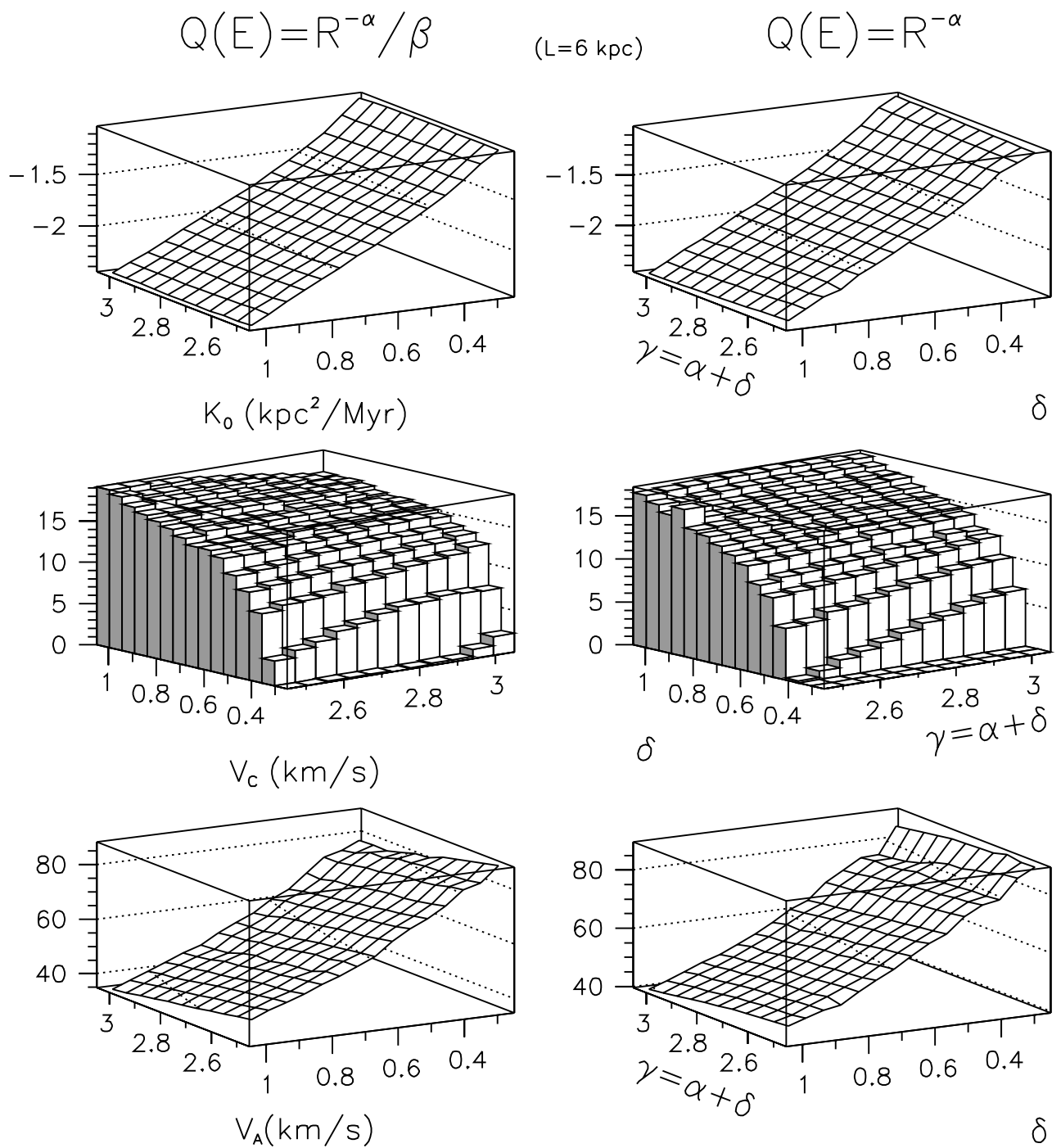

Fig. 9. From top to bottom: for each best $\chi^{2}$ in the plane $\delta-\gamma(L=6 \mathrm{kpc})$, the corresponding values of $\log \left(K_{0}\right), V_{\mathrm{c}}$ and $V_{\mathrm{a}}$ are plotted for both source spectrum types.

to compute the sub-Fe/Fe ratio, for each value of $\alpha$ and $\delta$ (for type (a) spectra and $L=6 \mathrm{kpc}$, although the results for type (b) and/or different $L$ are quite similar). This surface is very similar to the surface obtained with $B / C$, pointing towards high values of $\delta$ (compare to Fig. 8).

\subsubsection{Looking for $\chi_{\mathrm{Fe}}^{2}$}

We now consider a full sub-Fe/Fe analysis (i.e. the parameters minimizing $\chi_{\mathrm{Fe}}^{2}$ are looked for) but we emphasize that the results given here are from our point of view far less robust than those obtained from $B / C$. As a consequence, conclusions of this section have to be taken only as possible trends. Several points can be underlined from Fig. 12: (i) as for the $B / C$ case, the best $\chi_{\mathrm{Fe}}^{2}$ is obtained for type (b) spectra. (ii) the general behavior of $K_{0}, V_{\mathrm{c}}$ and to a less extent $V_{\mathrm{a}}$ is mostly the same as for $B / C$. (iii) the type (b) spectra yield propagation parameters which are closer to $B / C$ 's, as we can see from $V_{\mathrm{c}}$ values; (iv) finally, consistency with $B / C$ analysis would be better obtained for $\delta$ pointing towards $0.6-0.7$.

\subsection{Additional insight from visual comparison of our model to data}

Typical spectra (modulated at $\phi=500 \mathrm{MV}$ ) are shown in Fig. 13, for different values of the parameters $\alpha$ and $\delta$, along with the data points from HEAO-3 (Engelmann et al. 1990) and balloon flights (Dwyer \& Meyer 1987). Three low-energy data points, from HET on Ulysses (Duvernois \& Thayer 1996), нKH on ISEE-3 (Leske 1993) and Voyager (Webber et al. 2002) are also shown; they all have about the same modulation parameter, i.e. $\phi \approx 500 \mathrm{MV}$. The ACE points $(\phi \approx 750 \mathrm{MV})$ are also displayed (Davis et al. 2002).

All the models displayed give similar spectra, which would be difficult to sort by eye. This may explain why some of these models (e.g. those with $\delta=0.3$ ) are retained in other studies. The main features are (i) the influence of $\delta$ on the high energy behaviour - a good discrimination between these models would be provided by precise measurements around $100 \mathrm{GeV} / \mathrm{nuc}-$ and (ii) the type (a) source spectra are steeper than type (b) at low energy. 

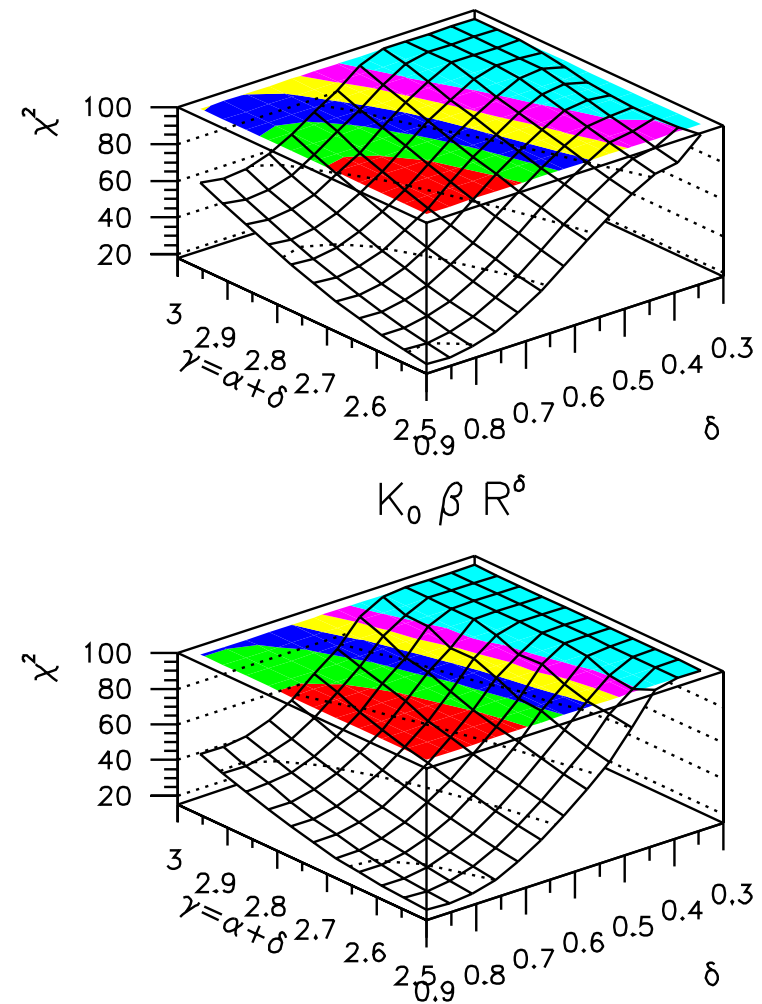

$K_{0} \beta^{2-\delta} R^{\delta}$

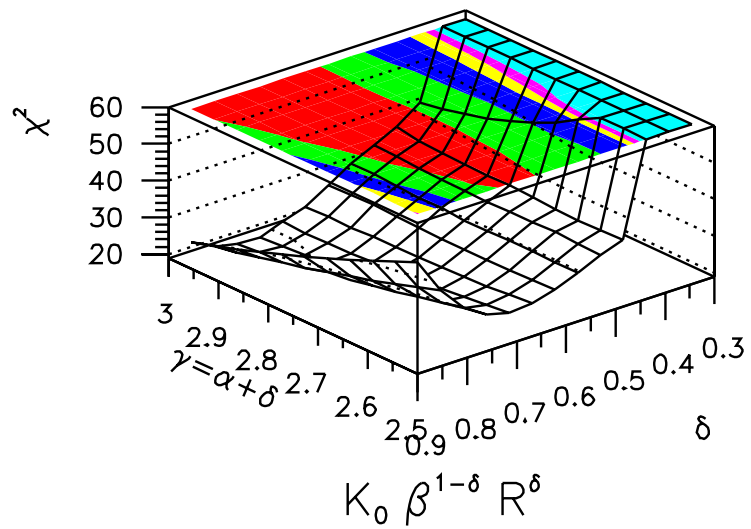

Fig. 10. Best $\chi^{2}$ values, in the plane $\delta-\gamma$, for the three different forms of the diffusion coefficient and reacceleration terms (i) Slab Alfven wave turbulence, with $K_{\mathrm{A}}(p)=K_{0} \beta \mathcal{R}^{\delta}$ and $K_{\mathrm{pp}}^{\mathrm{A}} \propto$ $V_{\mathrm{A}}^{2} p^{2} / K_{\mathrm{A}}(p)$, (ii) Isotropic fast magnetosonic wave turbulence, with $K_{\mathrm{F}}(p)=K_{0} \beta^{2-\delta} \mathcal{R}^{\delta}$ and $K_{\mathrm{pp}}^{\mathrm{F}} \propto V_{\mathrm{A}}^{2} p^{2} \beta^{1-\delta} \ln \left(v / V_{\mathrm{A}}\right) / K_{\mathrm{F}}(p)$, and (iii) mixture of the two last cases, $K_{\mathrm{M}}(p)=K_{0} \beta^{1-\delta} \mathcal{R}^{\delta}$ and $K_{\mathrm{pp}}^{\mathrm{M}}=K_{\mathrm{pp}}^{\mathrm{F}}$.

\section{Comparison with other works}

Some of our configurations can be compared to those previously found in similar models. In particular, to compare the Alfvén speed from one paper to another, we have to be sure that all $V_{\mathrm{a}}$ used denote the same quantity.

To compare the reacceleration terms employed, we retain only the spallation term and the highest order derivative in energy in the diffusion equation, giving

$2 h n_{\mathrm{ISM}} v \sigma_{j} N_{j}(0)=2 h \beta^{2} K_{\mathrm{pp}} \frac{\partial^{2} N_{j}(0)}{\partial E^{2}}$.

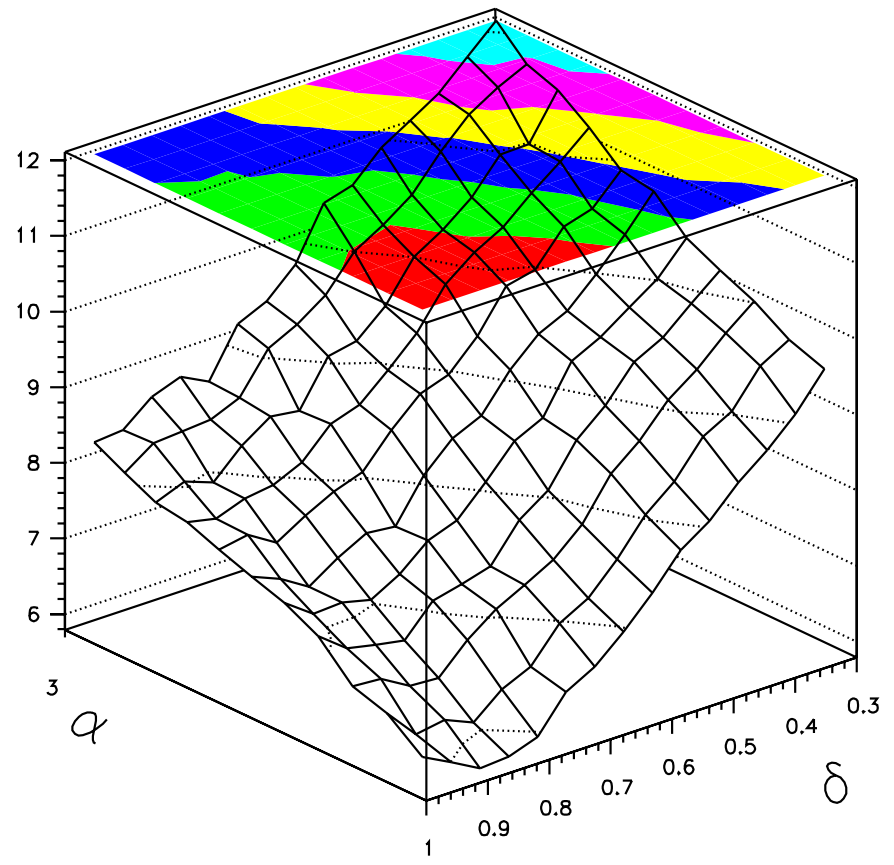

Fig. 11. Values of $\chi_{B / C \rightarrow \mathrm{Fe}}^{2}$ obtained by applying the diffusion parameters - type (a) source spectra and $L=6 \mathrm{kpc}$ - giving the best fit to $B / C$, for each $\alpha$ and $\delta$, to sub-Fe/Fe. The $\chi_{B / C \rightarrow \mathrm{Fe}}^{2}$ are computed with HEAO-3 data points.

We have supposed that both phenomena occur only in the thin disk $h \ll L$ and, in the above equation, the reacceleration zone height equals the spallative zone height. If it is not the case, we have to correct the previous relation by a multiplying factor $h_{\text {reac }} / h$. Actually, $V_{\mathrm{a}}$ is "fixed" through the choice of $K_{\mathrm{pp}} K(E)$. As underlined in Sect. 3.1, this paper now follows the requisites of minimal reacceleration models (see Table 1, last line).

Once this $h_{\text {reac }} / h_{\text {gas }}$ rescaling - that differs from one paper to another - is taken into account, a comparison is possible between models if a minimal resemblance exists between the other input parameters, i.e. same $\delta, \alpha$ (plus same form of the source spectrum) and halo size $L$; Table 1 shows the value adopted for these parameters in two recent studies.

\subsection{Maurin et al. (2001) - Paper I}

The results are expected to be slightly different from our previous study as the components have been modified. First, $V_{\mathrm{a}}$ has a different interpretation in the two studies (see Table 1, first column). As underlined above - remembering that in Paper I the diffusion coefficients scaled as $K_{\mathrm{pp}} K(E) \equiv(2 / 9) \times p^{2} V_{\mathrm{a}}^{2}-$, the Alfvén speed value from Paper I ( $\left.V_{\mathrm{a}}^{\text {Paper I }}\right)$ has to be rescaled into $V_{\mathrm{a}}^{\text {Paper I, Standard }}$ (i.e. as the standard convention used in this work and others) through the relation

$V_{\mathrm{a}}^{\text {Paper I }}=V_{\mathrm{a}}^{\text {Paper I, Standard }} \sqrt{\frac{6}{\delta\left(4-\delta^{2}\right)(4-\delta)}}$.

Second, the equation describing diffusion in energy has been modified and, the values of $K_{0}, V_{\mathrm{c}}$ and $V_{\mathrm{a}}$ that give the best fit to $B / C$ data for a given $\delta$ must change at some level. Notice 


$$
Q(E)=R^{\alpha} / \beta \quad(L=6 k p c) \quad Q(E)=R^{\alpha}
$$
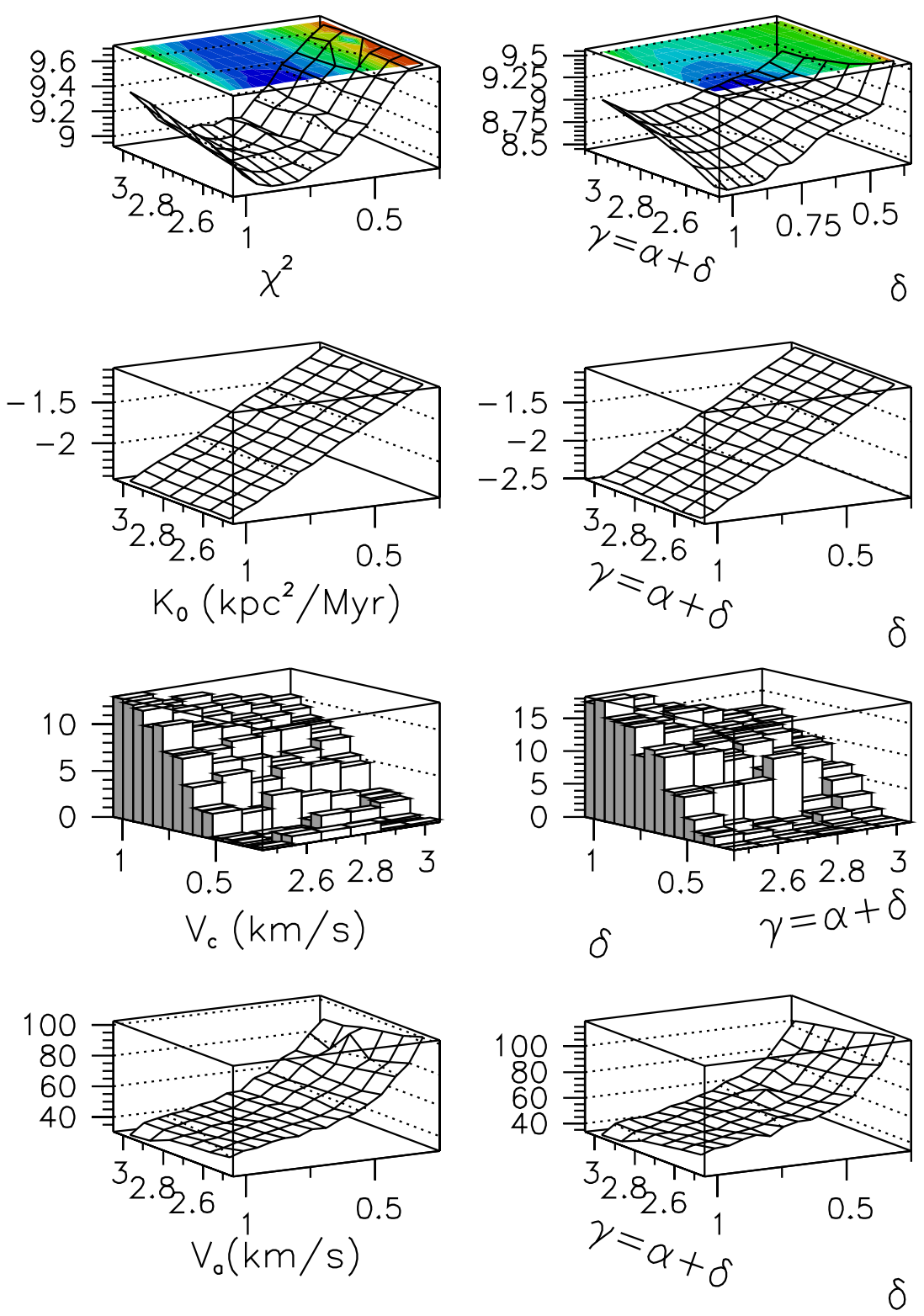

Fig. 12. From top to bottom: best $\chi_{\mathrm{Fe}}^{2}$ and for each best $\chi_{\mathrm{Fe}}^{2}$ in the $\delta-\gamma$ plane $(L=6 \mathrm{kpc})$, the corresponding values of $\log \left(K_{0}\right), V_{\mathrm{c}}$ and $V_{\mathrm{a}}$ are plotted for both source spectrum types.

that in Paper I we used a source term corresponding to type (b) spectra (see Eq. (11)), with $\gamma$ of each species that were set to their measured value (see details in Paper I); this corresponds roughly to $\gamma \approx 2.7$ for all boron progenitors.

However, we find that the conclusions raised in Paper I, in particular the behaviors reflected in Figs. 7 and 8 of Paper I, are basically unchanged (it is not straightforward to compare with present figures, but the careful reader can check this result using the above scaling relation and the corresponding parameter combinations). To be more precise, it appears that $K_{0} / L$ does not significantly change (for example, for $\delta=0.6$ and $L=2 \mathrm{kpc}$, we still have $K_{0} / L \sim 0.004 \mathrm{kpc} \mathrm{Myr}^{-1}$, see Fig. 3 left panel - this paper - and Fig. 7 of Paper I). As regards the galactic convective wind, $V_{\mathrm{c}}$ is shifted towards higher values, whereas the $V_{\mathrm{a}} / \sqrt{K_{0}}$ range remains roughly unchanged.

This can be easily understood: the additional term - comparable to a first order gain in energy, see Eq. (7) - has to be balanced to keep the fit good. This balance is ensured by enhanced adiabatic losses, i.e. bigger $V_{\mathrm{c}}$. Other parameters are only very slightly affected by this new balance.

\subsection{Jones et al. (2001); Moskalenko et al. (2002)}

Moskalenko et al. (2002) (hereafter Mos02) use a description more refined than ours because they include a realistic gas distribution. Jones et al. (2001) (hereafter Jon01) take advantage 

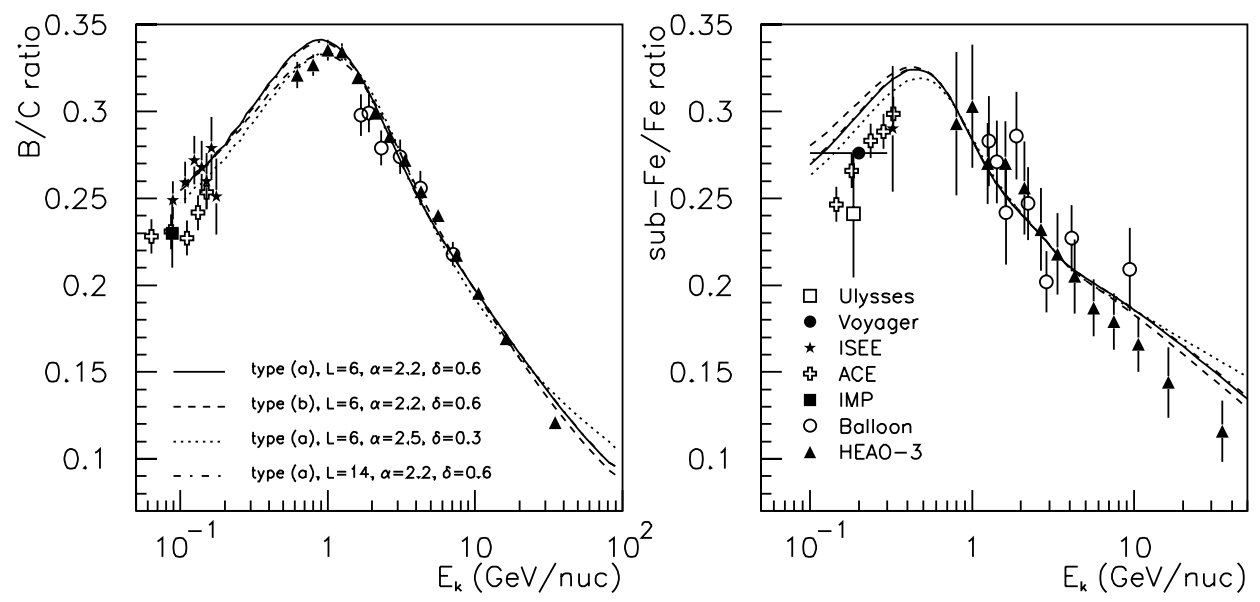

Fig. 13. The $B / C$ and sub-Fe/Fe spectra (modulated at $\phi=500 \mathrm{MV}$ ) for several sets of parameters (giving the best fit to $B / C$ for these values) are displayed, along with experimental data from HEAO-3 (Engelmann et al. 1990), ballon flights (Dwyer \& Meyer 1987), HET on Ulysses (Duvernois \& Thayer 1996), HKH on ISEE-3 (Leske 1993) and Voyager (Webber et al. 2002). Note that ACE data (Davis et al. 2002) correspond to a modulation parameter $\phi \approx 750 \mathrm{MV}$.

Table 1. Main characteristics of various diffusion models.

\begin{tabular}{cccc}
\hline \hline & Maurin et al./This work & Seo \& Ptuskin/Jones et al. & Moskalenko et al. \\
& $(2001) /(2002)$ & $(1994) /(2001)$ & $(2002)$ \\
\hline Thin disk $h(\mathrm{pc})$ & $h \equiv 100 \mathrm{pc}$ & $h=200 \mathrm{pc}$ & Gas distribution \\
Halo size $L(\mathrm{kpc})$ & - & $L=3 \mathrm{kpc}$ & $L=4 \mathrm{kpc}$ \\
$2 h_{\text {reac }}$ & $2 h_{\text {reac }}=2 h$ & $2 h_{\text {reac }}=2 L / 3^{\dagger}$ & $2 h_{\text {reac }}=2 L$ \\
Surface mass density & $\simeq 10^{-3} \mathrm{~g} \mathrm{~cm}^{-2}$ & $\simeq 2.0 \times 10^{-3} / 2.4 \times 10^{-3} \mathrm{~g} \mathrm{~cm}^{-2}$ & $\simeq 1.6 \times 10^{-3} \mathrm{~g} \mathrm{~cm}^{-2}$ \\
$K_{\mathrm{pp}} K(E)$ & $\frac{2 p^{2} V_{\mathrm{a}}^{2}}{9} / \frac{4 p^{2} V_{\mathrm{a}}^{2}}{3 \delta\left(4-\delta^{2}\right)(4-\delta) w}$ & $\frac{4 p^{2} V_{\mathrm{a}}^{2}}{3 \delta\left(4-\delta^{2}\right)(4-\delta) w}$ & $\frac{4 p^{2} V_{\mathrm{a}}^{2}}{3 \delta\left(4-\delta^{2}\right)(4-\delta) w}$ \\
\hline
\end{tabular}

Jones et al. use the same set of equation and parameters as Seo and Ptuskin for the stochastic reacceleration model. Consequently, it seems that $h_{\mathrm{a}}$ defined in Jones et al. is the half-height of the reacceleration zone, contrarily to what is depicted in their Fig. 4.

$\ddagger$ The surface mass density is defined as $\mu=2 h \rho$ where $\rho$ is the matter density in the thin disk.

of an equivalent description in terms of a leaky box formalism (use of a phenomenological diffusion coefficient) - for both wind model (no reacceleration) and minimal reacceleration model (no wind) - to solve the diffusion equation.

Let us make a few comments at the qualitative level. First, starting with Mos02's models, we can note that convection has always been disfavored by these authors. For example, in their first paper of a series (Strong \& Moskalenko 1998), a gradient of convection greater than $7 \mathrm{~km} \mathrm{~s}^{-1} \mathrm{kpc}^{-1}$ was excluded. We notice that this result was not very convincing since it is clear from an examination of their figures that none of the models they proposed gave good fits to $B / C$ data. Thanks to many updates in their code, their fits were greatly improved (Strong \& Moskalenko 2001; Moskalenko et al. 2002) but if it is now qualitatively good, it is hard to say how good it is since no quantitative criterion is furnished. Anyway, our Fig. 2 allows us to understand why convection is disfavored in such models. Actually, if $\delta \sim 0.3$ - as in the Kolmogorov diffusion slope hypothesis $\delta=1 / 3-$, we see that for such a configuration, the best fits are obtained for $V_{\mathrm{c}} \sim 0 \mathrm{~km} \mathrm{~s}^{-1}$.

Similar comments apply to Jon01's models. Given a Kolmogorov spectral index for the diffusion coefficient, their combined fit to $B / C$ plus sub-Fe/Fe data is not entirely satisfactory. It improves for higher values of $\delta$ and in the convective model (they do not include reacceleration in this model), their best fit being obtained for $\delta=0.74$. As the authors emphasized, the search in parameter space was not automated and they cannot guarantee that their best fit is the absolute best fit. Actually, the sub-Fe/Fe contribution to the $\chi^{2}$ value has to be taken with care. First, the error bars are not estimated well enough to give a statistical meaning for $\chi^{2}$ values (see Sect. 4.2) and a different weight should be considered for $B / C$ and sub-Fe/Fe. Second, if the best parameters extracted from $B / C$ data reproduce formally the same $\chi^{2}$ surface when applied to the evaluation of sub-Fe/Fe (see Fig. 11), the direct search for the parameters minimizing $\chi^{2}$ for the same sub-Fe/Fe data gives constraints that are much weaker (see Fig. 12). Thus, any conclusion including this ratio is from our point of view far less robust.

\subsection{Quantitative comparison, interpretation of $K_{0}, V_{c}$ and $V_{a}$}

\subsubsection{Justification of the differences between models}

Tables 2 and 3 give the results of Mos02 and Jon01 - without any rescaling of any parameters - compared to what is obtained here; only a few models are displayed. 
Table 2. Diffusion parameters obtained in models with $\delta=0.30, \alpha=2.40$ for pure power-law source spectra.

\begin{tabular}{ccccccccc}
\hline \hline $\begin{array}{c}L \\
(\mathrm{kpc})\end{array}$ & $\begin{array}{c}h \\
(\mathrm{kpc})\end{array}$ & $\begin{array}{c}\mu \times 10^{-3} \\
\left(\mathrm{~g} \mathrm{~cm}^{-2}\right)\end{array}$ & $\begin{array}{c}h_{\text {reac }} \\
(\mathrm{kpc})\end{array}$ & $\begin{array}{c}K_{0} \\
\left(\mathrm{kpc}^{2} \mathrm{Myr}^{-1}\right)\end{array}$ & $\begin{array}{c}V_{\mathrm{c}} \\
\left(\mathrm{km} \mathrm{s}^{-1}\right)\end{array}$ & $\begin{array}{c}V_{\mathrm{a}} \\
\left(\mathrm{km} \mathrm{s}^{-1}\right)\end{array}$ & $\chi_{\mathrm{r}}^{2}$ & Ref. \\
\hline 4. & $n(r)$ & 1.6 & 4.0 & $\sim 0.201$ & 0. & 30. & Good & Mos02 $^{\S}$ \\
3. & 0.2 & 2.4 & 1.0 & $\sim 0.196$ & 0. & 40. & 1.8 & $\begin{array}{c}\text { Jon01 }^{\dagger} \\
3 .\end{array}$ \\
0.1 & 1.0 & 0.1 & $\sim 0.0535$ & 0. & 105.8 & 4.2 & ${\text { (Figs. 8 and 9, this paper })^{\ddagger}}^{\text {This work }^{\ddagger}}$ \\
\hline 3. & 0.2 & 2.4 & 1.0 & $\sim 0.127$ & 0. & 47.3 & 4.4 & This $^{+}$
\end{tabular}

$\S$ For this model, the exact values are $\delta=0.33, \alpha=2.43$.

$\dagger$ This model give best fit to flux using a slightly modified form for the source; $Q \propto R^{-2.40} /\left[1-(R / 2)^{-2}\right]^{1 / 2}$.

$¥$ Corresponds to the best fit for the presented $L, \alpha$ and $\delta$ value.

Table 2 shows $K_{0}, V_{\mathrm{c}}$ and $V_{\mathrm{a}}$ for $\delta=0.30$ and $\alpha=2.40$. Taking the first three lines at face value, our values of $K_{0}$ and $V_{\mathrm{a}}$ are very different from the others and our model seems to have a problem. However, the matter disk properties (height and surface density) are different in these models. To be able to compare, we set these quantities to the values given in Jon01 and the resulting parameters are shown in the last line of Table 2.

Actually, we know that in diffusion models, the behavior is driven by the location of the closer edge, leading to a preferred escape on this side. With $L=3 \mathrm{kpc}$, our three-dimensional model should behave as the two-dimensional model with infinite extension in the $r$ direction of Jon01. This hypothesis can be validated if one takes their Eq. (3.6). For the pure diffusion model (reacceleration and convection are discarded), one has a simple relation between $\mu, L$ and $K_{0}$ through an equivalent leaky box grammage

$X_{\text {dif }}=\frac{\mu v L}{2 K_{0} R^{\delta}}$.

A direct application of this result to our model with $h=100 \mathrm{pc}$ (third line of Table 2) using the scaling $\mu \rightarrow 2.4 \times \mu$, leads to a rescaling $K_{0} \rightarrow 2.4 \times K_{0}$, consistent with results of the fourth line.

A similar expression may be obtained in the presence of galactic wind: in the wind model (their Eq. (4.6)), one has

$X_{\mathrm{w}}=\frac{\mu v}{2 V_{\mathrm{c}}}\left[1-\exp \left(\frac{-V_{\mathrm{c}} L}{K_{0} R^{\delta}}\right)\right]$.

Applied to the second line of Table 3, this gives $V_{\mathrm{c}} \rightarrow 2.4 \times V_{\mathrm{c}}$, leading in turn to $K_{0} \rightarrow 2.4 \times K_{0}$, also in very good agreement with the direct output of our code.

Even with this $\mu$ rescaling, the diffusion coefficients obtained by the different authors quoted above are still not fully compatible. Another possible effect, namely the spatial distribution of cosmic ray sources, is now investigated. We note that in our model, the radial distribution of sources $q(r)$ follows the distribution of supernovæ and pulsar remnants. The choice of this distribution has an effect on $B / C$ spectra and on the parameters giving the best fits. If we use a constant source distribution $q(r)=$ cte with $V_{\mathrm{a}}$ set to 0 to follow Jon01, we find that $K_{0}$ is enhanced by about $10 \%$. We checked that it is also the case for results presented in Table 2. Hence, it appears that results for $\delta=0.74$ of Jon01, though slightly different, are not in conflict with ours. As regards $\delta=0.3$ and Mos2, using the scaling relation (16) along with a $10 \%$ decrease of $K_{0}$ for Jon01, we obtain respectively $K_{0}=0.226(\mathrm{Mos} 02), 0.176$ (Jon01), 0.127 (this paper) $\mathrm{kpc}^{2} \mathrm{Myr}^{-1}$. Thus there is some difference between Mos02 and Jon01, which is not obvious when the values taken naively from Table 2 are compared. These discrepencies could have several origins: treatment of cross-sections (we checked that total and spallative cross sections - taking into account ghost nuclei, see Paper I - are compatible with recent data, e.g. Korejwo et al. 2002), average surface density in Mos02 that is probably not exactly 1.6, choice of data and fits for Jon01 that differ from ours (some of the point they used are significantly lower than HEAO-3's). Finally, the fact that we scan the whole parameter space can make a difference from manual search. To conclude, results are qualitatively similar, but a few quantitative differences remain. The intrinsic complications and subtleties of the various propagation codes make it difficult to go further in the analysis of these differences.

\subsubsection{Meaning of $K_{0}$}

The normalization $K_{0}$ gives a measure of the efficiency of the diffusion process at a given energy. Its value can be predicted if (i) a good modelling of charged particles in a stochastic magnetic field and (ii) a good description of the actual spatial structure of this magnetic field, were available. It is not the case and the precise value of $K_{0}$ is of little interest. Moreover, the presence of effects other than pure diffusion can be mimicked, at least to some extent, by a change in $K_{0}$. Equation (17) gives a whole class of parameters giving the same results and can be used to extract an effective value of $K_{0}$ taking into account the effect of the size of the halo $L$ and wind $V_{c}$. This also explains the great range of values that can be found in the literature.

This relation shows that there is also an indeterminacy of the absolute density of the model, because as long as $h \times n_{\text {ISM }}$ is constant, the grammage $X_{\text {dif }}$ is also constant. Fortunately, a realistic distribution of gas can be deduced by more direct observational methods, so that a definite value of $n_{\text {ISM }}$ can be used.

\subsubsection{Galactic convective wind $V_{\mathrm{c}}$}

We note that in our model, Galactic wind is perpendicular to the disk plane and is constant with $z$. Actually, the exact form of galactic winds is not known. From a self-consistent analytical description including magnetohydrodynamic calculations 
Table 3. Diffusion parameters obtained in models $\delta=0.74, \alpha=2.35$.

\begin{tabular}{ccccccccc}
\hline \hline $\begin{array}{c}L \\
(\mathrm{kpc})\end{array}$ & $\begin{array}{c}h \\
(\mathrm{kpc})\end{array}$ & $\begin{array}{c}\mu \times 10^{-3} \\
\left(\mathrm{~g} \mathrm{~cm}^{-2}\right)\end{array}$ & $\begin{array}{c}h_{\text {reac }} \\
(\mathrm{kpc})\end{array}$ & $\begin{array}{c}K_{0} \\
\left(\mathrm{kpc}^{2} \mathrm{Myr}^{-1}\right)\end{array}$ & $\begin{array}{c}V_{\mathrm{c}} \\
\left(\mathrm{km} \mathrm{s}^{-1}\right)\end{array}$ & $\begin{array}{c}V_{\mathrm{a}} \\
\left(\mathrm{km} \mathrm{s}^{-1}\right)\end{array}$ & $\chi_{\mathrm{r}}^{2}$ & Ref. \\
\hline 3. & 0.2 & 2.4 & 1.0 & $\sim 0.024$ & 29. & 0. & 1.5 & Jon01 \\
3. & 0.1 & 1.0 & 0.1 & $\sim 0.0056$ & 15.5 & 35.3 & 3.0 & This work \\
\hline 3. & 0.2 & 2.4 & 0.1 & $\sim 0.0134$ & 36.5 & 26.5 & 3.1 & - \\
\hline
\end{tabular}

$\stackrel{+}{*}$ Corresponds to the best fit for the presented $L, \alpha$ and $\delta$ value.

of the galactic wind flow, cosmic-ray pressure and the thermal gas in a rotating galaxy, Ptuskin et al. (1997) (see also references therein) find a wind increasing linearly with $z$ up to $z \sim 15 \mathrm{kpc}$, with a $z=0$ value of about $22.5 \mathrm{~km} \mathrm{~s}^{-1}$. Following a completely different approach, Soutoul \& Ptuskin (2001) extract the velocity form able to reproduce data from a one-dimensional diffusion/convection model. They obtain a decrease from $35 \mathrm{~km} \mathrm{~s}^{-1}$ to $12 \mathrm{~km} \mathrm{~s}^{-1}$ for $z$ ranging from $40 \mathrm{pc}$ to $1 \mathrm{kpc}$ followed by an increase to $20 \mathrm{~km} \mathrm{~s}^{-1}$ at about $3 \mathrm{kpc}$. For reference, our values for the best fits correspond to about $15 \mathrm{~km} \mathrm{~s}^{-1}$. The difficulty to compare constant wind values to other $z$-dependences is related to the fact that cosmic rays do not spend the same amount of time at all $z$, so that there cannot be a simple correspondence (see also next section) from one model to another. As a result, all the above-mentioned models are formally different, with different inputs (spectral index, diffusion slope). Nevertheless, their values are roughly compatible, Ptuskin et al's model providing the grounds for a physical motivation for this wind. However, an even more complicated form of the Galactic wind could be relevant for a global description of the Galaxy (see Breitschwerdt et al. 2002).

\subsubsection{Interpretation of the Alfvénic speed $V_{a}$}

Above, we gave some elements to compare $V_{\text {a }}$ values from various works. Actually, secondary to primary ratios are not determined directly by the Alfvén speed in the interstellar medium, but rather by an effective value:

$V_{\mathrm{a}}^{\text {true }}=\sqrt{\frac{h_{\text {reac }}}{h}} \times \frac{V_{\mathrm{a}}^{\mathrm{eff}}}{\sqrt{\omega}}$.

First, the parameter $\omega$ characterizes the level of turbulence and is often set to 1 (Seo \& Ptuskin 1994). Our model, as others, uses

$\omega(z)= \begin{cases}1 & \text { if } z<h_{\text {reac }}, \\ 0 & \text { otherwise }\end{cases}$

as a crude approximation of the more complex reality.

Second, the total rate of reacceleration (at least in a first approximation, see discussion below) is given by a convolution of the time spend in the reacceleration zone and the corresponding true Alfvén speed in this zone. There is a direct analogy with the case of spallations and the determination of the true density in the disk, as discussed above. The problem is still somewhat different, as there are no direct observational clues about the size of the reacceleration zone, or said differently, about $\omega(z)$. This leads to a degeneracy in $h / h_{\text {reac }}$ that holds as far as $h_{\text {reac }} \ll L$, due to the structure of the equations in the thin disk approximation. For example, a model such as Strong et al's that uses $h_{\text {reac }}=L$ cannot be simply scaled to ours. A cosmic ray undergoing reacceleration at a certain height $z$ has a finite probability of escaping before it reaches Earth, this probability being greater for greater $z$. As a result, the total reacceleration undergone by a cosmic ray is actually not a simple convolution of the reacceleration zone times the Alfvén speed in this zone, but rather should be an average along $z$ taking into account the above-mentioned probability (in principle, this remark also holds for the gaseous disk, though the latter is known to be very thin, $\sim$ a few hundreds of pc).

To conclude, there are basically three steps associated with three levels of approximations to go from the $V_{\mathrm{a}}$ deduced from cosmic ray analysis to the physical quantity. First, if $\omega(z)$ is approximatively constant with $z$, how large is the reacceleration zone height? The second level is related to the possibility that $\omega(z)$ strongly depends on $z$ in a large reacceleration zone. If it is too large, the link with the phenomenologically equivalent quantity in a thin zone is related to the vertical occupation of cosmic rays. However, this latter possibility seems to be unfavoured by MHD simulations (see Ptuskin et al. 1997). Finally, with the above parallel between interpretation of $\mu$ and $V_{\mathrm{a}}$, we see how misleading it is to obtain precise physical quantities from our simple model, since there is no one-to-one correspondence between reality and simplified models. This discussion shows that even if the actual derived Alfvén speeds are consistent with what is expected from "direct" observation $(\sim 10$ $30 \mathrm{~km} \mathrm{~s}^{-1}$ ), the cosmic ray studies would certainly be not very helpful in providing physical quantities better than a factor of two. If we reverse the reasoning and retain our best models with $L=6 \mathrm{kpc}$, we could conclude that $\sqrt{h / h_{\text {reac }}}$ must be $\sim 4$ in order to give realistic values for $V_{\mathrm{a}}$ (with evident a priori about $\omega(z)$ ).

\subsubsection{The evolution of propagation models}

As suggested by the previous discussion, there are several propagation schemes, each associated with numerous configurations, that are able to explain the $B / C$ data. Thus, the discussion should not be about the correctness of all these models (leaky boxes, two or three-dimensional diffusion models and their inner degeneracy), but rather about their domain of validity. As a matter of fact, they are all equivalent, as far as stable cosmic rays around $\mathrm{GeV} /$ nuc energies are considered.

Starting with the leaky box; it has been shown more than thirty years ago (Jones 1970) that the concept of "leakagelifetime" was appropriate for the charged nuclei considered 
here (see also Jones et al. 1989), even if it broke down for $\mathrm{e}^{-}$ (all orders in the development in "leakage" eigenmodes contribute because of synchrotron or inverse Compton losses) and for radioactive nuclei (Prishchep \& Ptuskin 1975). The leaky box, due to its simplicity, is very well suited for the extraction of source abundances (elemental as well as isotopic). It can also be used for secondary antiproton production, since the same processes as for secondary stable nuclei are at work. However, as emphasized in Paper II, leaky box models are not able to predict any primary contribution in the antiproton signal, since it requires the knowledge of the spatial distribution of primary progenitors. Considering a possible extension of leaky box models for stable charged nuclei to high energy $(\sim \mathrm{PeV})$, it has been demonstrated in Maurin et al. (2002) that they are to a good approximation sufficient to describe the evolution of cosmic rays. Last, it is well known that leaky box parameters are just phenomenological with only a distant connection to physical quantities.

This was further realized by Jones $(1978,1979)$ who first remarked that the phenomenological behavior of the escape length at low energy could be due to the presence of a Galactic wind. Jones et al. (2001) investigated further this idea and generated several equivalent phenomenological escape lengths from several possible physical configurations of a one-dimensional diffusion model. The relation between onedimensional models and leaky box models is thus firmly established and very well understood. Moreover, this relation elucidates some of the physical content of leaky box models. Now if one wishes to overcome the inherent limitations of these models and say, to compute some primary antiproton component, one has to go through a three-dimensional model. It is likely that these models can also be related to the Jones et al. models (see Taillet \& Maurin, in preparation). Several arguments used in the previous sections illustrate this view, but this occurs at least if the halo size is small compared to radial extension of the Galaxy.

In the semi-analytical two-zone model used here, it is possible to evaluate the primary antiproton component (see Barrau et al. 2001) and to take into account radioactive species, even in the presence of a local very underdense bubble (see Donato et al. 2002 for details). Our model fails to consider species such as $\mathrm{e}^{-}$and $\mathrm{e}^{+}$, since the latter suffer from large energetic losses in the halo so that no simple semi-analytical approach can be used. The parameters extracted from these models are much easier to interpret in terms of physical quantities.

Most of the limitations mentionned above are overcome by Strong et al's models. In this fully numerical model, all cosmic ray species can be computed self-consistently with the same propagation parameters. The main difference with our model is that a more realistic matter distribution is used instead of a thin homogeneous disk. They also consider that reacceleration occurs in the whole diffusion halo, which in our opinion is an approximation no more justified than the fact to confine it in a thin disk (see discussion in Sect. 6.3.4). Considering the gas distribution, both models are equally predictive for the charged nuclei (including antiprotons, see Fig. 9 of Paper II). On the one hand, our approach is better suited to scan the whole parameter space as we did in Paper I and in this paper. On the other hand, the Strong et al. models can check the consistency of $\mathrm{e}^{-}, \mathrm{e}^{+}$and $\gamma$ with observations, and can include whatever deviation from ideal cases for $K_{0}, V_{\mathrm{a}}, V_{\mathrm{c}}$ and more generally for any ingredient that enters in the description of propagation models.

To conclude about models and their use, Jones et al.'s approach is probably the best and simpliest way to understand how physical parameters affect the propagated flux. Our model is very well suited for a consistent evaluation of all charged nuclei and extraction of propagation parameters; furthermore it is an intermediate step where general behaviors can still be analytically explored (Taillet \& Maurin, in preparation). In the Strong et al. model, all fine effects can be studied and modelled, with the counterpart that the numerical approach makes the physical intuition of the results less straightforward. In its present form, Strong et al's model can be viewed as a fully numerical version of ours, so that their behaviors are very close. This discussion could leave the reader with a feeling that apart from these different modellings left to personal taste, galactic propagation phenomena are well understood. It is surely not the case! Even if all these models are equivalent to describe the local observations of charged cosmic rays, they lead to very different conclusions and interpretations when the spatial variation of the cosmic ray density is considered. As an illustration of the poor current understanding of this global aspect, we mention the ever-lasting problem of the gamma ray excess about $1 \mathrm{GeV}$ towards the Galactic center or the too flat radial $\gamma$-ray distribution observed in the disk (see Breitschwerdt et al. 2002).

\section{Conclusion}

Forgetting for a while some of our theoretical a priori about the diffusion power spectrum, a new picture of cosmic ray propagation seems to emerge, motivated by the $B / C$ analysis. In this new picture, high values for the diffusion coefficient spectral index $(\delta \gtrsim 0.6-0.7)$ and source spectral indices $\alpha \sim 2.0$ are favored. This latter result is rather satisfactory: as emphasized in a recent working group report on SNR shocks (Drury et al. 2001), even "if nonlinear acceleration models do not produce precise power-law spectra [...] the effective differential energy spectral index is close to 2.0." Furthermore, as pointed out in a series of papers by Vainio $\mathcal{E}$ Schlickeiser (2001, and see references therein), diffusive shock wave acceleration naturally yields smaller values of $\alpha$ if the correct scattering center compression ratio is used instead of the gas compression ratio.

This trend should be carefully analysed and discussed in the light of measured differential fluxes, in order to confirm or point out the possible inconsistencies in the current propagation treatments (see companion paper, Donato et al. in preparation). Briefly, the major arguments against large $\delta$ come from anisotropy measurements at high energy and from theoretical preference for Kolmogorov-like turbulence spectra. However, Ptuskin et al. (1997) - in their self-consistent analytical propagation model including gas, cosmic ray and magnetic field - derived $\delta \sim 0.55, \alpha \sim 2.1$ and argue that the observed anisotropy could be as well due to a particularity of the local structure of the Galactic magnetic field. Theoretical objections against too high values of $\delta$ are probably more robust. 
For the rest, the conclusions of this paper can be summarized as follows: (i) we performed for the first time a full analysis of diffusion/convection/reacceleration models in the whole 6-dimensional parameter space $\left(\alpha, \delta, K_{0}, L, V_{\mathrm{a}}, V_{\mathrm{c}}\right)$, and the values $\delta \sim 0.7-0.9$ and $\alpha \sim 2.0$ are preferred; (ii) this preference holds whatever the specific form of the spectrum at low energy; the numerical values of the other parameters are also only slightly modified by this low energy dependence even though deviation from a power-law at low energy is preferred. The study of fluxes should give a more definite answer; (iii) $K_{0}$ scales logarithmically with $\delta$ and models with small halos tend to one-dimensional models with a simple relation between $\mu, K_{0}, L$ and $V_{\mathrm{c}}$ (see also Taillet \& Maurin, in preparation); (iv) several existing models are compared and the qualitative and quantitative differences between them are studied and partially explained.

Acknowledgements. D.M. would like to thank Aimé Soutoul for valuable remarks and Michel Cassé and Elisabeth Vangioni-Flam for many interesting discussions. We thank Prof. Schlickeiser for providing us with the diffusion coefficients in the different schemes in a useful form.

\section{References}

Barrau, A., Boudoul, G., Donato, F., et al. 2002, A\&A, 388, 676

Berezhko, E. G., Yelshin, V. K., \& Ksenofontov, L. T. 1994, Astropart. Phys., 2, 215

Berezinskii, V. S., Bulanov, S. V., Dogiel, V. A., Ginzburg, V. L., \& Ptuskin, V. S. 1990, Astrophysics of Cosmic Rays (Amsterdam: North-Holland)

Breitschwerd, D., Dogiel, V. A., \& Völk, H. J. 2002, A\&A, 385, 216

Davis, A. J., et al. 2000, Proc. ACE-2000 Symp., ed. Mewaldt, Jokipii, Lee, Möbius \& Zurbuchen, AIP Conf. Proc., 528, 421
Donato, F., Maurin, D., \& Taillet, R. 2002, A\&A, 381, 539

Donato, F., Maurin, D., Salati, P., et al. 2001, ApJ, 563, 172 (Paper II)

Drury, L. O'C., et al. 2001, Space Sci. Rev., 99, 329

Duvernois, M. A., \& Thayer, M. R. 1996, ApJ, 465, 982

Dwyer, R., \& Meyer, P. 1987, ApJ, 322, 981

Engelmann, J. J., Goret, P., Juliusson, E., et al. 1985, A\&A, 148, 12

Engelmann, J. J., et al. 1990, A\&A, 233, 96

Gieseler, U. D. J., Jones, T. W., \& Kang, H. 2000, A\&A, 364, 911

Jones, F. C. 1970, Phys. Rev. D, 12, 2787

Jones, F. C. 1978, ApJ, 222, 1097

Jones, F. C. 1979, ApJ, 229, 747

Jones, F. C., Lukasiak, A., Ptuskin, V., \& Webber, W. 2001, ApJ, 547, 264

Jones, F. C., et al. 1989, Proceedings of the 21st ICRC, OG 8.2-1, vol. 3, 333

Korejwo, A., Giller, M., Dzikowski, T., Perelygin, V. V., \& Zarubin, A. V. 2002, J. Phys. G: Nucl. Part. Phys., 28, 1199

Leske, R. A. 1993, ApJ, 405, 567

Maurin, D., Donato, F., Taillet, R., \& Salati, P. 2001, ApJ, 555, 585 (Paper I)

Maurin, D., Cassé, M., \& Vangioni-Flam, E. 2002, Astropart. Phys., accepted [astro-ph/0202334]

Moskalenko, I. V., Strong, A. W., Ormes, J. F., \& Potgieter, M. S. 2002, ApJ, 565, 280

Prishchep, V. L., \& Ptuskin, V. S. 1975, Ap\&SS, 32, 265

Ptuskin, V. S., Völk, H. J., Zirakashvili, V. N., \& Breitschwerdt, D. 1997, A\&A, 321, 434

Schlickeiser, R. 2002, Cosmic Ray Astrophysics (Springer-Verlag)

Seo, E. S., \& Ptuskin, V. S. 1994, ApJ, 431, 705

Soutoul, A., \& Ptuskin, V. S. 2001, ICRC 27, OG 3.1.16

Strong, A. W., \& Moskalenko, I. V. 1998, ApJ, 509, 212

Strong, A. W., \& Moskalenko, I. V. 2001, Adv. Space Res., 27, 717

Vainio, R., \& Schlickeiser, R. 2001, A\&A, 378, 309

Webber, W. R., Lukasiak, A., \& McDonald, F. B. 2002, ApJ, 568, 210

Wiebel-Sooth, B., Biermann, P. L., \& Meyer, H. 1998, A\&A, 330, 389 